\title{
The Identification of a New Species, Diaporthe humulicola, a Pathogen Causing Diaporthe Leaf Spot on Common Hop
}

\author{
Elisha Allan-Perkins, ${ }^{1}$ De-Wei Li, ${ }^{1}$ Neil Schultes, ${ }^{2}$ Sumeyra Yavuz, ${ }^{2}$ and James LaMondia ${ }^{1, \dagger}$ \\ ${ }^{1}$ Valley Laboratory, The Connecticut Agricultural Experiment Station, Windsor, CT, 06095-0248, U.S.A. \\ ${ }^{2}$ Plant Pathology and Ecology, The Connecticut Agricultural Experiment Station, New Haven, CT, 06504-1106, U.S.A.
}

\begin{abstract}
Common hop, Humulus lupulus, is a commercially important crop in the United States, with an increasing number of hop yards being established in the Northeast. In 2018, a new fungal disease was observed at two research hop yards in Connecticut. This new pathogen affected all hop cultivars being grown and caused leaf spots and browning of cones. The causal organism was isolated and Koch's postulates were performed to confirm pathogenicity. The disease symptoms were similar to the previously described Phoma wilt; however, morphological and phylogenetic analyses placed the causal organism as a new species of Diaporthe. We propose the name Diaporthe humulicola. The disease increased under

hot, humid conditions (around $24^{\circ} \mathrm{C}$ and $90 \%$ relative humidity), which prevail during the summer in the northeastern United States as well as other parts of the country. An in vitro preliminary assessment of fungicide sensitivity revealed that pyraclostrobin and boscalid inhibited $D$. humulicola growth in culture and should be further assessed for field efficacy against this new disease of hop. The proper identification and monitoring of this pathogen will be important to inform hop growers of this new threat.

Keywords: Coelomycetes, Diaporthaceae, Humulus lupulus, new pathogen, Phomopsis
\end{abstract}

Common hop, Humulus lupulus L., is grown commercially for production of hop cones used predominately for preservation and flavoring in beer and, to a lesser extent, for personal care products and some medicines (Mahaffee et al. 2009; Stevens and Page 2004; Zanoli and Zavatti 2008). The United States leads the world in hop production with acreage primarily in the Pacific Northwest region, including Oregon, Washington, and Idaho (George 2018). In the past 10 years, there has been a resurgence in hop cultivation in the northeastern United States to meet the demand for local ingredients from the growing craft brew industry and requirements for labeling as a local product. The Connecticut Agricultural Experiment Station initiated a hop research project in 2013 that consisted of creating two hop yards, one in Windsor, CT and one in Hamden, CT, to be managed similarly to commercial hop. Each year since plant establishment, hop plants were evaluated for presence and severity of fungal diseases and insect pests (Allan-Perkins et al. 2019b). Common diseases present in the Northeast have been similar to the Pacific Northwest. We have found that the most prevalent disease in this region is downy mildew. Powdery mildew is an equally destructive hop disease (Mahaffee et al. 2009) but has only been reported from one hop yard in Windsor, CT (J. LaMondia, personal communication) and one hop yard in Colchester, CT (Allan-Perkins et al. 2019a).

In July and August 2018, lesions were detected on hop leaves at both of the Connecticut Agricultural Experiment Station hop yards. Leaf spot symptoms were ellipsoid, brownish-gray lesions, often with white rings, sometimes with chlorotic margins (Figs. 1A and B). Leaf spot lesions were not delimited by veins and often were

${ }^{\dagger}$ Corresponding author: J. LaMondia; james.lamondia@ct.gov

Funding: United States Department of Agriculture Agricultural Marketing Service Grant Number 16-SCBGP-CT-0012.

*The $\boldsymbol{e}$-Xtra logo stands for "electronic extra" and indicates that one supplementary figure and one supplementary table are published online.

The author(s) declare no conflict of interest.

Accepted for publication 24 March 2020.

C 2020 The American Phytopathological Society associated with leaf margins (Figs. 1A and B). Lesions were detected on eight cultivars: Fuggle, Willamette, Hallertauer, Chinook, Galena, AlphAroma, Perle, Newport, Saaz, and Summit. On bines of the cultivar Saaz, cones presented with brown margins on the bracts in addition to infected leaves (Fig. 1C). Symptoms were similar to those described for Phoma wilt, caused by Phoma exigua Desm. (Mahaffee et al. 2009). Infected leaves had dark pycnidia producing ellipsoid eguttulate conidia, similar to that of $P$. exigua (Mahaffee et al. 2009). This pathogen has been reported to cause disease on hop in China, Europe, and New Zealand (Mahaffee et al. 2009; Radisek et al. 2008). Metagenomic analysis of hop plants in Slovenia found Phoma spp. present on symptomatic and asymptomatic plants, along with other fungal species such as Alternaria, Fusarium, and Sclerotinia spp. (Jakse et al. 2015). In Canada, Phoma spp. are considered occasional pathogens on hop (Grant and Filotas 2014). Within the United States, Phoma spp. have been reported as causing secondary infection of hop cones, leading to browning, in Vermont (Darby 2017).

P. exigua, synonym of Boeremia exigua var. exigua, has previously been called Phyllosticta decidua Ellis \& Kellerm. 1883, which was reported as causing disease on hop in Wisconsin in 1944 (Greene 1944) and in Iowa in 1929 (Gilman and Archer 1929; USDA 1960). Phoma herbarum was reported on hop in Spain and China (Farr and Rossman 2019; Gonzalez Fragoso 1917; Zhuang 2005). Other Phoma spp. reported as causing infection on hop are $P$. aliena (syn. Didymella aliena) in the Netherlands (Boerema et al. 2004) and P. macrostoma in Poland (Farr and Rossman 2019; Mulenko et al. 2008).

The objective of this study was to determine the identity of the Phoma-like pathogen causing disease on hop in Connecticut. Morphological identification as well as molecular analyses were used to determine the identity of the fungus. Koch's postulates were performed to confirm the pathogenicity of the isolated fungus on hop leaves. Fungicide efficacy was tested for three fungicides registered for use on hop in Connecticut that have been reported to have efficacy for Phoma and Phomopsis spp. on other crops. The results of this study will provide important information for hop growers on diseases present in the northeastern United States and potential control strategies.

\section{Materials and Methods}

Field identification, collection, and culturing. Hop bines planted in Windsor, CT at the Connecticut Agricultural Experiment Station 
Valley Laboratory were observed to have lesions on leaves on 31 July 2018. Leaves from cultivars AlphAroma, Newport, Saaz, Willamette, Fuggle, Galena, and Chinook and a cone from the Saaz plant were collected and brought into the laboratory. The edge of one lesion from each leaf or cone was excised from the tissue, surface sterilized in $10 \%$ bleach solution for $30 \mathrm{~s}$ followed by a rinse for $30 \mathrm{~s}$ in sterile water, and plated onto half-strength potato dextrose agar (1/2PDA). Cultures were grown at $20^{\circ} \mathrm{C}$ with a cycle of $12 \mathrm{~h}$ of light and $12 \mathrm{~h}$ of darkness. Hyphal tip isolations were performed for each original culture on 1/2PDA. Conidial suspensions were made using sterile water to create streak plates for isolation on 1/2PDA. Plugs from hyphal tip isolations and solitary colonies from streak plates were transferred to $1 / 2 \mathrm{PDA}$ slants for long-term storage. Additional leaf samples were pressed for long-term storage and the remaining leaf samples were split and half frozen and half refrigerated for further use.

On 7 August 2018, similar lesions were observed on hop in Hamden, CT at the Connecticut Agricultural Experiment Station Lockwood Farm. All cultivars were affected. Leaves from cultivars AlphAroma, Newport, Cascade, Summit, Sterling, and Brewer's Gold were collected and brought to the laboratory. Leaves were processed as described for those collected from Windsor, CT. In May 2019, similar lesions were observed on hop in Windsor and Hamden, $\mathrm{CT}$ and leaves were processed as previously described. By the end of August, all cultivars displayed symptoms; however, Cascade bines showed the fewest symptoms. Hop yield loss due to disease was not assessed. Cascade and Newport yield increased at Windsor Farm from 2017 to 2018 but decreased at Lockwood Farm E. AllanPerkins, K. Mauer, and J. A. LaMondia, unpublished). Summit yield also increased at Windsor but cones were not collected at Lockwood Farm due to poor cone quality (E. Allan-Perkins, K. Mauer, and J. A. LaMondia, unpublished). AlphAroma yield was lower at Lockwood Farm in 2018 than 2017 and cones were not collected at Windsor farm due to poor quality (E. Allan-Perkins, K. Mauer, and J. A. LaMondia, unpublished).

Isolates from AlphAroma (leaf) in Windsor, Saaz (cone) in Windsor, and Newport (leaf) in Hamden were used for molecular identification and denoted as CT2018-1, CT2018-2, and CT2018-3, respectively. CT2018-1 was used as the holotype and identified morphologically. Plugs from fungal isolates CT2018-1 were placed individually in $1.5-\mathrm{ml}$ centrifuge tubes with $1 \mathrm{ml}$ of sterilized distilled water in triplicate and submitted to the UAMH Centre for Global Microfungal Biodiversity at the University of Toronto (UAMH Collection ID 12076). Temperature and precipitation data were collected by the Connecticut Agricultural Experiment Station weather stations (HOBO U30 with S-THB-M002 12 Bit Temp/RH Sensor and S-RGB-M002 Rain Gauge; Onset Computer Company, Bourne, MA, U.S.A.) in Windsor and Lockwood, CT. In early September, all hop cultivars at both locations showed symptoms with nearly $100 \%$ of bines affected.
Morphological identification. Leaf lesions were observed under an Olympus SZ11dissecting scope for presence of pycnidia (Fig. 2). Pycnidia were excised from the leaf lesion, soaked in 50\% ammonia solution for $4 \mathrm{~h}$, placed into $50 \%$ gum arabic solution, frozen using a BFS-3MP Freezing Stage (Physitemp Instruments LLC, Cliften, NJ, U.S.A.), and sliced with a Microm HM310 microtome (Thermo Scientific, Waltham, MA, U.S.A.) at $10-\mu \mathrm{m}$ thickness. Four sliced pycnidia were measured and photographed using an Axiocam 506 color camera mounted to a Zeiss Imager M2 compound microscope with differential interference contrast (Carl Zeiss AG, Oberkochen, Germany) with the Zeiss software ZEN (Fig. 2b). Additional pycnidia (Fig. 2c), conidiophores (Fig. 2d), and conidia (Figs. 2e and f) were measured using an Olympus BX40 compound microscope (Olympus Life Sciences, Tokyo, Japan) and an Olympus BH2 compound microscope, for a total of 30 of each from three different isolates. Means, standard deviations of the measurements, and 95\% confidence intervals of means were calculated using the Data Analysis package in Microsoft Excel for Mac (v. 16.16.13; Microsoft Corporation, Redmond, WA, U.S.A.).

DNA extraction, amplification, and sequencing. Genomic DNA was extracted from fungal cultures of isolates CT2018-1, CT2018-2, and CT2018-3 grown on 1/2PDA using the ZR Fungal/Bacterial DNA MicroPrep Kit (Zymo Research, Irvine, CA, U.S.A.) according to manufacturer's instructions. The resulting DNA was subject to PCR using oligonucleotides V9G or ITS5 with ITS4 or LR1 for the internal transcribed spacer (ITS) region of ribosomal DNA (Van den Ende and de Hoog 1999; Vilgalys and Hester 1990; White et al. 1990), EF1-728F and EF1-986R for translation elongation factor $\alpha-1(\mathrm{EF} 1 \alpha)$ (Carbone and Kohn 1999), CYLH3F and H3-1b for histone (HIS) (Crous et al. 2004; Glass and Donaldson 1995), CAL288F and CAL737R or CAL235F and CAL-2Rd for calmodulin (CAL) (Carbone and Kohn 1999; Groenewald et al. 2013; Quaedvlieg et al. 2012), LROR and LR7 for the large ribosomal subunit (28S) (Rehner and Samuels 1994; Vilgalys and Hester 1990), fRPB2-5F2 and fRPB2-7cR RNA polymerase II subunit (RBPS2) (Liu et al. 1999), and TUBUF2 and TUBUR1 (Kroon et al. 2004) for $\beta$-tubulin (TUB) amplification. The parameters for the PCR protocol were $94^{\circ} \mathrm{C}$ for $3 \mathrm{~min}, 94^{\circ} \mathrm{C}$ for $30 \mathrm{~s}, 45^{\circ} \mathrm{C}$ for $30 \mathrm{~s}$, and $72^{\circ} \mathrm{C}$ for 2 min, repeat 40 times; and $72^{\circ} \mathrm{C}$ for 7 min with $50^{\circ} \mathrm{C}$ as annealing temperature for the HIS PCR.

The resulting PCR products were purified using QIAquick PCR Purification columns (Qiagen, Valencia, CA, U.S.A.) and DNA concentrations were determined on a NanoDrop Lite Spectrophotometer (Thermo Scientific). The PCR products were sequenced using the following oligonucleotides: ITS by ITS1-5 and LR1 (Vilgalys and Hester 1990; White et al. 1990); LSU by LROR, LR5, LR3R, LR3B, and LR7 (Li et al. 2017; Vilgalys and Hester 1990; White et al. 1990), EF1 $\alpha$ by EF1-728F and EF1-986R; HIS by CYLH3F and H3-1b (Crous et al. 2004; Glass and Donaldson 1995), CAL
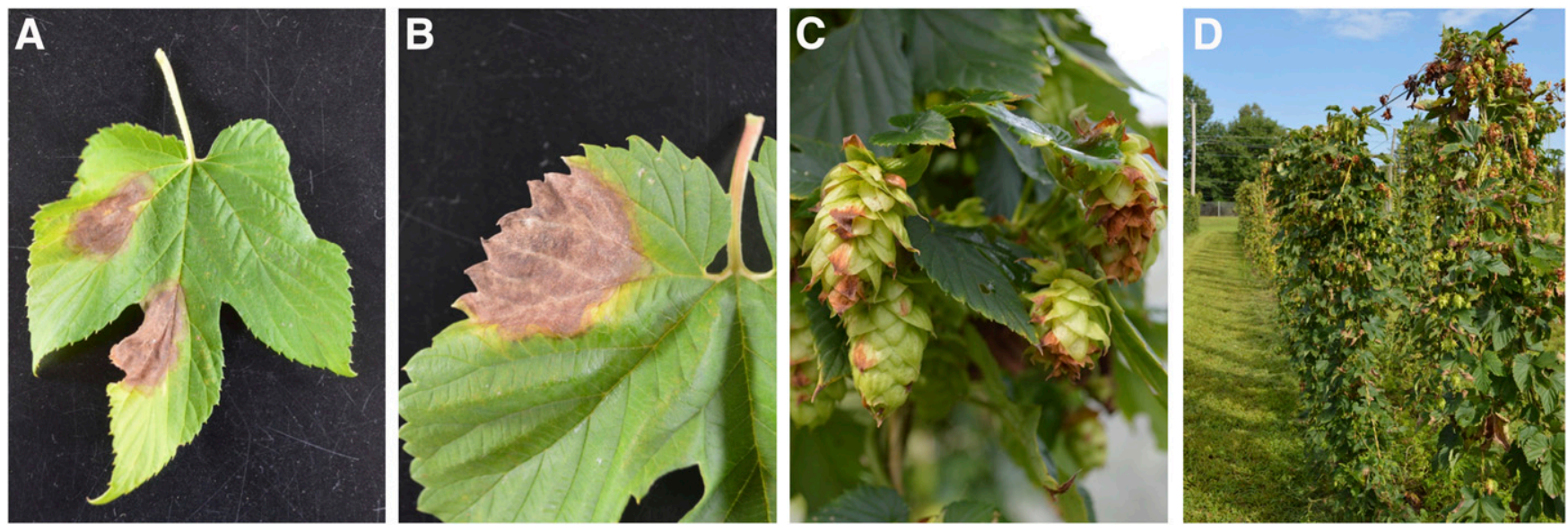

Fig. 1. Diaporthe leaf spot caused by Diaporthe humulicola on $\mathbf{A}$ and $\mathbf{B}$, leaf tissue; $\mathbf{C}$, cone tissue; $\mathbf{D}$, affected hop bines of common hop (Humulus lupulus) at Valley Laboratory Farm in Windsor, CT. 
by CAL288F, CAL737R, CAL235F, or CAL-2Rd (Carbone and Kohn 1999; Groenewald et al. 2013 Quaedvlieg et al. 2012); and $\beta$-tubulin by TUBUF1, TUBUR2, T12, and T22 (Kroon et al. 2004; O'Donnell and Cigelnik 1997). All DNA sequencing was performed at the W. M. Keck Biotechnology Resource Laboratory, Yale School of Medicine (New Haven, CT, U.S.A.). DNA sequence information was deposited to GenBank, accession numbers of the taxa and isolates that were newly sequenced in the study are listed in Table 1, and sequences are listed in Supplementary Table S1.

Alignment and phylogenetic analyses. Sequences for each gene were aligned using Molecular Evolutionary Genetics Analysis (MEGA-7) across computing platforms (Kumar et al. 2016). Pairwise distances were calculated among the isolates using the maximum-likelihood model. Sequences from the type specimens were compared against nucleotide sequences in the NCBI GenBank database using the nucleotide BLAST search algorithm.

DNA sequences were obtained from NCBI GenBank for the ITS region of ribosomal DNA for Phoma spp. previously reported as causing disease on hop, representative species of the genus Diaporthe, and additional members of the family Diaporthaceae (Table 2). These sequences were aligned with the ITS sequences for three isolates of the new putative species using ClustalW within MEGA-7. A phylogenetic tree was created using maximumlikelihood analysis with 1,000 bootstrap replicates in MEGA-7 (Fig. 3).

To better place the CT2018 fungal isolates, a five-gene tree was created using ITS, EF1 $\alpha$, TUB, HIS, and CAL. These loci had the most representative sequences in the NCBI GenBank for Diaporthe spp. Sequences used by Gomes et al. (2013) to resolve the Diaporthe phylogeny were downloaded from GenBank (Table 3) and aligned with the Connecticut isolates using the online program MAFFT (https://mafft.cbrc.jp/alignment/server) using default settings (Katoh and Toh 2008) for each locus individually. The alignments were manually edited to remove large gaps and to be of equal lengths for all loci using the program MEGA-7. The alignments were combined using the online interface FaBox (https://users-birc.au.dk/ palle/php/fabox/alignment_joiner.php) (Villesen 2007). Bayesian inference was analyzed for the combined dataset of ITS, EF1 $\alpha$, TUB, HIS, and CAL sequences with MrBayes3.2.6 (Ronquist et al. 2012). Four Markov chains were used for four runs from random starting trees for 2 million generations. Tree sampling frequency was 1,000 generations. The first $1 / 10$ of generations were discarded as burnin. A majority-rule consensus tree of all remaining trees was calculated. Branches that received Bayesian posterior probabilities of 0.95 were set as significantly supported. Phylogenetic trees were drawn with TreeGraph2 (Stöver and Müller 2010). Sequence

Table 1. GenBank accessions for Connecticut Diaporthe humulicola isolates

\begin{tabular}{llc}
\hline Isolate & \multicolumn{1}{c}{ Locus } & Accession number \\
\hline CT2018-1 & Calmodulin & MN180204 \\
CT2018-2 & Calmodulin & MN180205 \\
CT2018-3 & Calmodulin & MN180206 \\
CT2018-1 & Translation elongation factor 1 $\alpha$ & MN180207 \\
CT2018-2 & Translation elongation factor 1 $\alpha$ & MN180208 \\
CT2018-3 & Translation elongation factor 1 $\alpha$ & MN180209 \\
CT2018-2 & $\beta$ Tubulin & MN180210 \\
CT2018-1 & $\beta$ Tubulin & MN180211 \\
CT2018-3 & $\beta$ Tubulin & MN180212 \\
CT2018-1 & Histone 3 & MN180213 \\
CT2018-2 & Histone 3 & MN180214 \\
CT2018-3 & Histone 3 & MN180215 \\
CT2018-1 & RNA polymerase II subunit & MN180216 \\
CT2018-2 & RNA polymerase II subunit & MN180217 \\
CT2018-3 & RNA polymerase II subunit & MN180218 \\
CT2018-1 & Internal transcribed spacer & MN152927 \\
CT2018-2 & Internal transcribed spacer & MN152928 \\
CT2018-3 & Internal transcribed spacer & MN152929 \\
CT2018-1 & Large ribosomal subunit & MN152977 \\
CT2018-2 & Large ribosomal subunit & MN152978 \\
CT2018-3 & Large ribosomal subunit & MN152979 \\
\hline
\end{tabular}
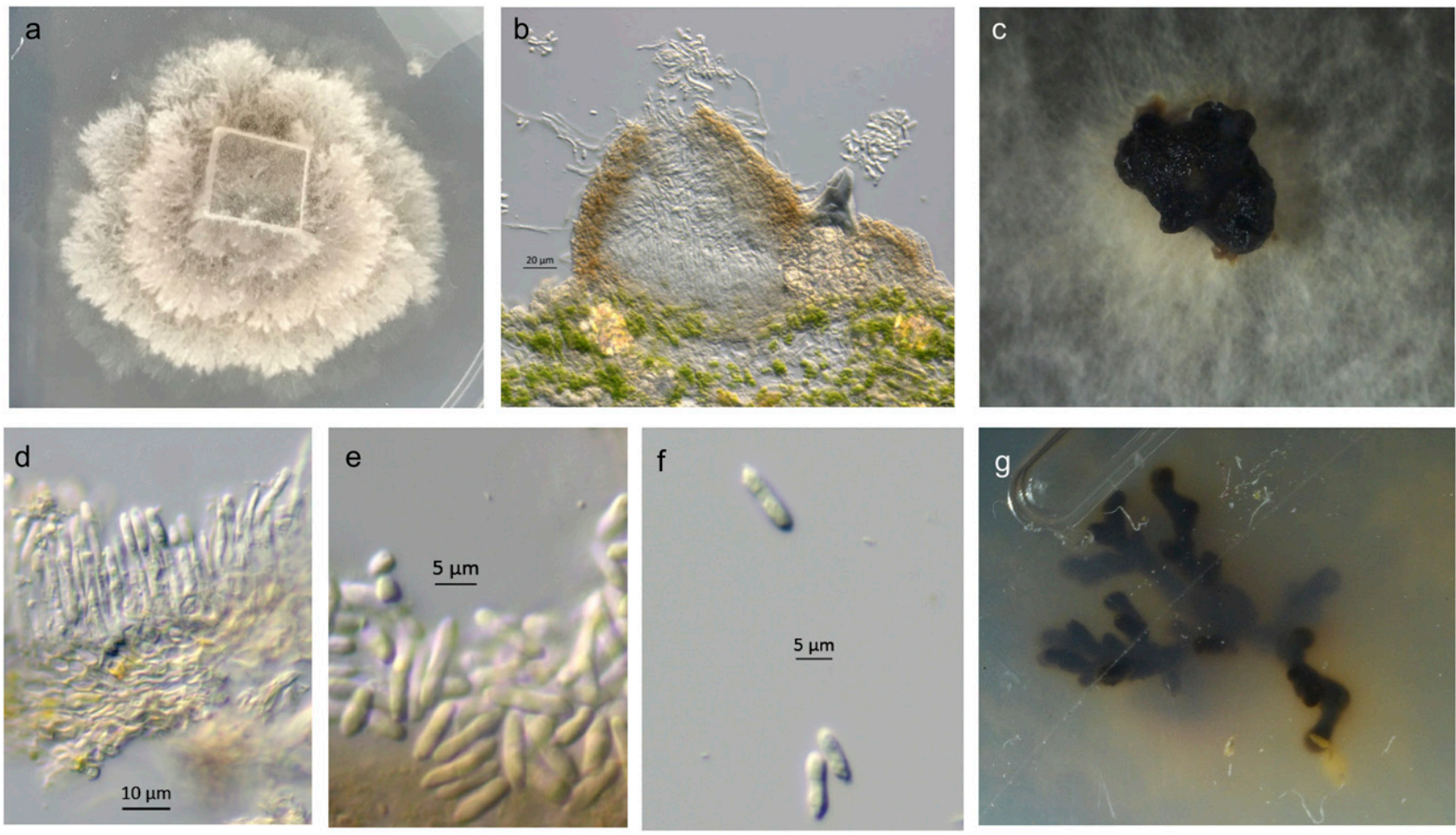

Fig. 2. Diaporthe humulicola (Holotype UAMH 12076) a, growing on half-strength potato dextrose agar (1/2PDA); b, a pycnidium on leaf tissue of common hop (Humulus lupulus); $\mathbf{c}$, pycnidium growing on 1/2PDA; $\mathbf{d}$, conidiophores lining the pycnidial wall of a pycnidium on leaf tissue of common hop; $\mathbf{e}$ and $\mathbf{f}$, conidia on an infected leaf of common hop; and $\mathbf{g}$, rhizomorph-like sclerotia in which a number of pycnidia were embedded produced by isolate CT2018-3. 
alignments for the genus placement and placement with the Diaporthe trees were submitted to TreeBASE and accessed online (https://treebase.org/treebase-web/search/study/anyObjectAsRDF.rdf? namespacedGUID=TB2:S25003).

Koch's postulates. Strap cuttings were taken from hop plants in June 2018 and maintained in the greenhouse with ambient light. In September 2018, conidia were harvested from the hyphal tip culture of the CT2018-1 isolate by adding approximately $1 \mathrm{ml}$ of sterile water to the plate and dislodging conidia with a sterile glass rod to make a conidial suspension. Then, $5 \mathrm{ml}$ of a conidial suspension $\left(2 \times 10^{6}\right.$ conidia/ml) was sprayed on each strap cutting for a total of six plants. Two negative control plants received $5 \mathrm{ml}$ of sterile water. The strap cuttings were then placed in clear plastic bags and evaluated for disease presence after 7 days. Leaf lesions were excised from infected

Table 2. Sequences of Phoma spp. and members of the order Diaporthales used in phylogenetic analysis for genus determination of Diaporthe humulicola

\begin{tabular}{|c|c|c|c|c|c|}
\hline Species & Isolate & Type & Substrate & ITS $^{\mathbf{a}}$ & Reference \\
\hline Воегетіа ехіgиа var. exigua & CBS 141361 & - & Veronica officialis & KY550229 & Michel et al. 2018 \\
\hline B. exigua var. exigua & CBS 431.74 & - & Solanum tuberosum & FJ427001 & Aveskamp et al. 2009 \\
\hline Diaporthe acaciarum & CBS 138862 & $\mathrm{~T}$ & Acacia tortilis & KP004460 & Crous et al. 2014 \\
\hline D. anacardii & CBS 720.97 & $\mathrm{~T}$ & Anacardium occidentale & $\mathrm{NR}_{111841}$ & Gomes et al. 2013 \\
\hline D. arecae & CBS 161.64 & $\mathrm{~T}$ & Areca саtechu & KC343032 & Gomes et al. 2013 \\
\hline D. betulina & CFCC 52560 & $\mathrm{~T}$ & Betula albo-sinensi & MH121495 & Yang et al. 2018b \\
\hline D. citri & CBS 135422 & $\mathrm{~T}$ & Citrus sp. & KC843311 & Udayanga et al. 2014b \\
\hline D. eres & AR5193, CBS:138594 & $\mathrm{T}$ & Ulmus laevis & KJ210529 & Udayanga et al. 2014a \\
\hline D. ganjae & CBS 180.91 & $\mathrm{~T}$ & Cannabis sativa & KC343112 & Gomes et al. 2013 \\
\hline D. inconspicua & CBS 133813 & $\mathrm{~T}$ & Maytenus ilicifolia & KC 343123 & Yang et al. 2017 \\
\hline D. nothofagi & BRIP 54801 & $\mathrm{~T}$ & Nothofagus cunninghamii & JX862530 & Tan et al. 2013 \\
\hline D. perjuncta & CBS 109745 & $\mathrm{~T}$ & Ulmus glabra & $\mathrm{NR}_{147527}$ & Gomes et al. 2013 \\
\hline D. ravennica & MFLUCC 15-0479 & $\mathrm{T}$ & Tamarix sp. & KU900335 & Thambugala et al. 2017 \\
\hline D. rosae & MFLUCC 17-2658 & $\mathrm{T}$ & Rosa sp. & MG828894 & Wanasinghe et al. 2018 \\
\hline D. sambucusii & CFCC 51986 & $\mathrm{~T}$ & Sambucus williamsii & KY852495 & Yang et al. 2018b \\
\hline D. terebinthifolii & CBS 133180; LGMF914; & $\mathrm{T}$ & Schinus terebinthifolius & KC343216 & Gomes et al. 2013 \\
\hline D. unshiuensis & $\begin{array}{l}\text { ZJUD 52, } \\
\text { CGMCC } 3.17569\end{array}$ & $\mathrm{~T}$ & Citrus unshiu & KJ490587 & Yang et al. 2018b \\
\hline D. velutina & LC 4421 & $\mathrm{~T}$ & Neolitsea sp. & KX986790 & Gao et al. 2017 \\
\hline D. garethjonesii & MFLUCC $12-0542 \mathrm{~A}$ & $\mathrm{~T}$ & - & KT459423 & Dissanayake et al. 2015 \\
\hline Diaporthella corylina & CBS 121124 & - & Corylus sp. & KC343004 & Gomes et al. 2013 \\
\hline Diaporthosporella cercidicola & CFCC 51994 & - & - & KY852492 & Yang et al. 2018a \\
\hline D. cercidicola & CFCC 51995 & - & - & KY852493 & Yang et al. 2018a \\
\hline D. cercidicola & CFCC 51996 & - & - & KY852494 & Yang et al. 2018a \\
\hline Diaporthostoma machili & CFCC 52100 & $\mathrm{~T}$ & Machilus leptophylla & MG682080 & Fan et al. 2018 \\
\hline D. machili & CFCC 52101 & - & M. leptophylla & MG682081 & Fan et al. 2018 \\
\hline Didymella macrostoma & KP 00116 & - & Pyrus communis & MG791816 & $\begin{array}{l}\text { K. T. K. Pham, R. Berghuis, and } \\
\text { M. Wenneker, unpublished }\end{array}$ \\
\hline Ophiodiaporthe cyatheae & HMH-2013 YMJ 1364 & $\mathrm{~T}$ & Cyathea lepifera & JX570889 & Fu et al. 2013 \\
\hline $\begin{array}{l}\text { Phaeocytostroma } \\
\text { megalosporum }\end{array}$ & CBS 284.65 & - & Oryza sativa & FR748045 & Lamprecht et al. 2011 \\
\hline P. ambiguum & CPC 17072 & - & Zea mays & FR748037 & Lamprecht et al. 2011 \\
\hline P. ambiguum & CPC 17071 & - & Z. mays & FR748036 & Lamprecht et al. 2011 \\
\hline$P$. plurivorum & CBS 113835 & - & Helianthus annus & FR748046 & Lamprecht et al. 2011 \\
\hline P. sacchari & CBS 275.34 & - & - & MH855512 & Vu et al. 2019 \\
\hline Phoma aliena & CBS 379.93 & - & Berberis sp. & GU237851 & Aveskamp et al. 2010 \\
\hline P. aliena & CBS 877.97 & - & Buxus sempervirens & GU237910 & Aveskamp et al. 2010 \\
\hline P. aliena & ICMP 6602 & - & Actinidia deliciosa & КТ309949 & P. R. Johnston and D. Park, unpublished \\
\hline P. ехigua & ICMP 15330 & - & - & EU573008 & Irinyi et al. 2009 \\
\hline P. еxigua & IHRB 2PEX & - & Humulus lupulus & EF136399 & Radisek et al. 2008 \\
\hline P. exigua var. exigua & CBS 431.742 & - & H. lupulus & EF136400 & Radisek et al. 2008 \\
\hline P. herbarum & EF68d & - & - & KT355016 & Unpublished \\
\hline P. herbarum & CBS 615.75 & $\mathrm{~T}$ & Rosa multiflora & FJ427022 & Aveskamp et al. 2009 \\
\hline P. macrostoma & IMI 299239 & - & H. lupulus & DQ474110 & $\begin{array}{l}\text { W. M. Pitt, K. L. Bailey, Y.-B. Fu, and } \\
\text { G. W. Peterson, unpublished }\end{array}$ \\
\hline P. macrostoma & ICMP 6803 & - & Lolium perenne & KT309987 & P. R. Johnston and D. Park, unpublished \\
\hline P. macrostoma & ICMP 7033 & - & Trifolium fragiferum & KT310027 & P. R. Johnston and D. Park, unpublished \\
\hline Phomopsis conorum & CBS 587.79 & - & $\begin{array}{l}\text { Pinus parviflora var. } \\
\text { pentaphylla }\end{array}$ & KC343153 & Gomes et al. 2013 \\
\hline Phomopsis emicis & BRIP 45089a & $\mathrm{T}$ & Emex australis & JF957784 & Udayanga et al. 2011 \\
\hline P. fukushii & BRIP 45089b & - & - & JQ619898 & Udayanga et al. 2012 \\
\hline P. fukushii & CBS 116953 & - & Pyrus pyrifolia & KC 343147 & Gomes et al. 2013 \\
\hline Phomopsis tuberivora & CBS 268.32 & $\mathrm{~T}$ & Solanum tuberosum & JF957785 & Udayanga et al. 2011 \\
\hline Pustulomyces bambusicola & MFLUCC 11-0436 & $\mathrm{T}$ & Bamboo & KF548664 & Dai et al. 2014 \\
\hline Stenocarpella macrospora & CBS 117560 & - & Z. mays & FR748048 & Lamprecht et al. 2011 \\
\hline S. maydis & CBS 117558 & - & Z. mays & FR748051 & Lamprecht et al. 2011 \\
\hline Valsa ambiens & CFCC 89894 & - & Pyrus sp. & KR045617 & Fan et al. 2014 \\
\hline
\end{tabular}

${ }^{a}$ GenBank accession number for internal transcribed spacer. 
plants and plated onto 1/2PDA and incubated at ambient laboratory conditions for 7 days. Cultures were identified morphologically.

Fungicide efficacy. In order to determine possible control measures for this pathogen in the field, the fungicides trifloxystrobin (Bayer Crop Science, Flint, NC, U.S.A.), pyraclostrobin and boscalid (Pageant Intrinsic; BASF, Morrisville, NC, U.S.A.), and pyraclostrobin (Insignia Intrinsic SC; BASF) were tested for their ability to inhibit $D$. humulicola growth in vitro. Trifloxystrobin and the pyraclostrobin and boscalid mix (as Pristine; BASF) are currently registered for use on hop and listed as providing control for Phoma and Phomopsis spp. on other crops. To determine whether the combination of boscalid and pyraclostrobin is needed to control the disease, we also included the pyraclostrobin-only fungicide, although it is not registered for use on hop. We calculated fungicide rates

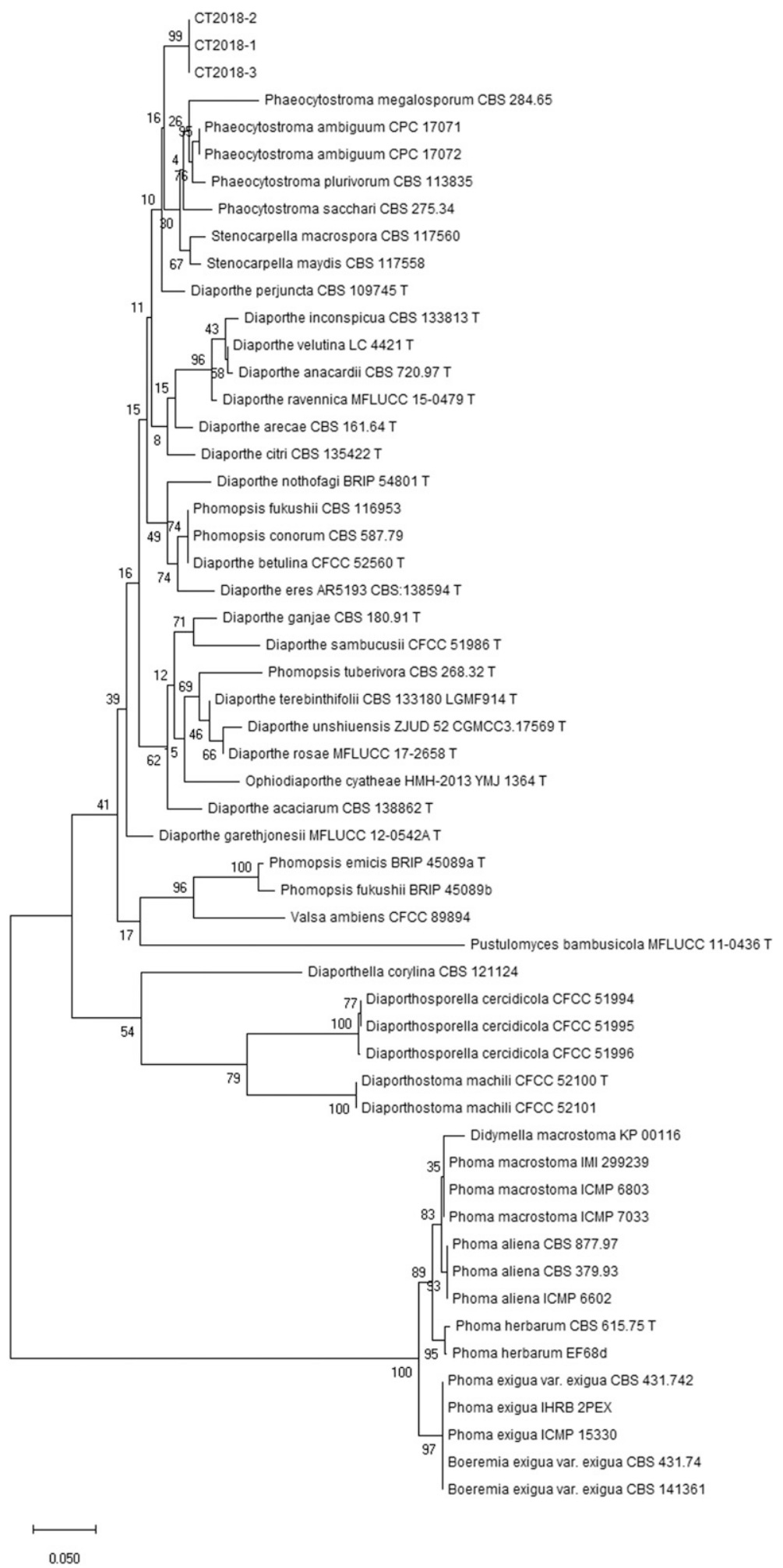

Fig. 3. Maximum-likelihood tree of Diaporthe humulicola isolates CT2018-1, CT2018-2, and CT2018-3 with members of Diaporthales and Phoma spp. using the internal transcribed spacer (ITS) unit locus of ribosomal DNA. Bootstrap values of resampling with 1,000 replicates are reported at the noses. 
Table 3. Sequences of Diaporthaceae species used in phylogenetic analysis (Gomes et al. 2013) for species placement of Diaporthe humulicola

\begin{tabular}{|c|c|c|c|c|c|c|}
\hline \multirow[b]{2}{*}{ Sequence name } & \multirow[b]{2}{*}{ Isolate } & \multicolumn{5}{|c|}{ GenBank accession numbers ${ }^{b}$} \\
\hline & & ITS & EF1 $\alpha$ & TUB & CAL & HIS \\
\hline D. acaciigena & CBS 129521; CPC $17622 \mathrm{~T}$ & KC343005 & KC343731 & KC343973 & KC343247 & КC343489 \\
\hline D. acerina & CBS 137.27 & KC343006 & KC343732 & KC343974 & KC343248 & КC343490 \\
\hline D. alleghaniensis & CBS 495.72; ATCC $24097 \mathrm{~T}$ & KC343007 & KC343733 & KC343975 & KC343249 & КC343491 \\
\hline D. alnea & CBS 146.46 & KC343008 & KC343734 & KC343976 & $\mathrm{KC} 343250$ & KC343492 \\
\hline D. alnea & CBS 159.47 & KC343009 & KC343735 & KC343977 & KC343251 & KC343493 \\
\hline D. ambigua & CBS 114015; STE-U 2657; CPC 2657 T & KC343010 & KC343736 & KC343978 & KC343252 & KC343494 \\
\hline D. ambigua & CBS 117167; STE-U 5414; CPC 5414 & KC343011 & KC343737 & KC343979 & KC343253 & KC343495 \\
\hline D. ambigua & CBS 127746; IMI 395956 & KC343014 & KC343740 & KC343982 & KC343256 & KC343498 \\
\hline D. ampelina & CBS 111888; ATCC 48153; STE-U 2673; CPC 2673 & KC343016 & KC343742 & KC343984 & KC343258 & KC343500 \\
\hline D. ampelina & CBS 114016; STE-U 2660; CPC 2660; PV F98-1 T & AF230751 & AY745056 & JX275452 & AY745026 & - \\
\hline D. ampelina & CBS 114867; STE-U 4708; CPC 4708 & KC343017 & KC343743 & KC343985 & KC343259 & KC343501 \\
\hline D. ampelina & CBS 267.80; STE-U 2671; CPC 2671 & KC343018 & KC343744 & KC343986 & KC343260 & KC343502 \\
\hline D. amygdali & CBS 111811; STE-U 2632; CPC 2632 & KC343019 & KC343745 & KC343987 & KC343261 & KC343503 \\
\hline D. amygdali & CBS 115620; FAU 1005 & KC343020 & KC343746 & KC343988 & KC343262 & KC343504 \\
\hline D. amygdali & CBS $126679 \mathrm{~T}$ & KC343022 & KC343748 & KC343990 & KC343264 & KC343506 \\
\hline D. amygdali & CBS 126680 & KC343023 & KC343749 & KC343991 & KC343265 & КC343507 \\
\hline D. arctii & CBS 136.25 & KC343031 & KC343757 & KC343999 & KC343273 & КC343515 \\
\hline D. arecae & CBS $161.64 \mathrm{~T}$ & KC343032 & KC343758 & KC344000 & KC343274 & KC343516 \\
\hline D. arecae & CBS 535.75 & KC343033 & KC343759 & KC344001 & KC343275 & KC343517 \\
\hline D. arengae & CBS 114979; HKUCC 5527 T & KC343034 & KC343760 & $\mathrm{KC} 344002$ & KC343276 & КC343518 \\
\hline D. aspalathi & CBS 117168; STE-U 5420; CPC 5420 & KC343035 & KC343761 & KC344003 & KC343277 & КC343519 \\
\hline D. aspalathi & CBS 117169; STE-U 5428; CPC 5428 T & KC343036 & KC343762 & KC344004 & KC343278 & KC343520 \\
\hline D. aspalathi & CBS 117500; STE-U 5408; CPC 5408 & KC343037 & KC343763 & KC344005 & KC343279 & KC343521 \\
\hline D. australafricana & CBS 111886; STE-U 2676; CPC 2676 T & KC343038 & KC343764 & KC344006 & KC343280 & KC343522 \\
\hline D. australafricana & CBS 113487; STE-U 2655; CPC 2655 & KC343039 & KC343765 & KC344007 & KC343281 & KC343523 \\
\hline D. batatas & CBS 122.21 & KC343040 & KC343766 & KC344008 & KC343282 & KC343524 \\
\hline D. beckhausii & CBS 138.27 & KC343041 & KC343767 & KC344009 & KC343283 & KC343525 \\
\hline D. brasiliensis & CBS 133183; LGMF924; CPC 20300 T & KC343042 & KC343768 & KC344010 & KC343284 & КC343526 \\
\hline D. brasiliensis & LGMF926; CPC 20302 & KC343043 & KC343769 & KC344011 & KC343285 & KC343527 \\
\hline D. carpini & CBS 114437; UPSC 2980 & KC343044 & KC343770 & $\mathrm{KC} 344012$ & KC343286 & КC343528 \\
\hline D. caulivora & CBS 127268; Dpc1 T & KC343045 & KC343771 & KC344013 & KC343287 & KC343529 \\
\hline D. caulivora & CBS 178.55; ATCC 12048; Alfaro 243 & KC343046 & KC343772 & KC344014 & KC343288 & KC343530 \\
\hline D. celastrina & CBS 139.27 & KC343047 & KC343773 & KC344015 & KC343289 & KC343531 \\
\hline D. chamaeropis & CBS 454.81 & KC343048 & KC343774 & KC344016 & KC343290 & KC343532 \\
\hline D. chamaeropis & CBS 753.70 & KC343049 & KC343775 & KC344017 & KC343291 & KC343533 \\
\hline D. cinerascens & CBS 719.96 & KC343050 & KC343776 & KC344018 & KC343292 & KC343534 \\
\hline D. citri & CBS 199.39 & KC343051 & KC343777 & KC344019 & KC343293 & КC343535 \\
\hline D. citri & CBS 230.52 & KC343052 & KC343778 & KC 344020 & KC343294 & KC343536 \\
\hline D. citri & LGMF946; CPC 20322 & KC343053 & KC343779 & KC344021 & KC343295 & KC343537 \\
\hline D. convolvuli & CBS 124654; DP 0727 & KC343054 & KC343780 & KC344022 & KC343296 & KC343538 \\
\hline D. crataegi & CBS 114435; UPSC 2938 & $\mathrm{KC} 343055$ & KC343781 & KC344023 & KC343297 & KC343539 \\
\hline D. crotalariae & CBS 162.33 & KC343056 & KC343782 & KC344024 & KC343298 & KC343540 \\
\hline D. cuppatea & CBS 117499; STE-U 5431; CPC 5431 T & KC343057 & KC343783 & KC344025 & KC343299 & KC343541 \\
\hline D. cynaroidis & CBS 122676; CMW 22190; CPC 13180 T & KC343058 & KC343784 & KC344026 & KC343300 & KC343542 \\
\hline D. decedens & CBS 109772; AR 3459 & KC343059 & KC343785 & KC344027 & KC343301 & KC343543 \\
\hline D. decedens & CBS 114281; UPSC 2957 & KC343060 & KC343786 & KC344028 & KC343302 & KC343544 \\
\hline D. detrusa & CBS 109770; AR 3424 & KC343061 & KC343787 & KC344029 & KC343303 & KC343545 \\
\hline D. detrusa & CBS 114652; UPSC 3371 & KC343062 & KC343788 & KC344030 & KC343304 & KC343546 \\
\hline D. detrusa & CBS 140.27 & KC343063 & KC343789 & KC344031 & KC343305 & KC343547 \\
\hline D. elaeagni & CBS 504.72 & KC343064 & KC343790 & KC344032 & KC343306 & KC343548 \\
\hline D. endophytica & CBS 133811; LGMF916; CPC 20292 T & KC343065 & KC343791 & KC344033 & KC343307 & KC343549 \\
\hline D. endophytica & LGMF911; CPC 20287 & KC343066 & KC343792 & KC344034 & KC343308 & KC343550 \\
\hline D. endophytica & LGMF919; CPC 20295 & KC343067 & KC343793 & KC344035 & KC343309 & KC343551 \\
\hline D. eres & CBS 375.61 & KC343088 & KC343814 & KC344056 & KC343330 & KC343572 \\
\hline D. eres & CBS 422.50 & KC343089 & KC343815 & KC344057 & KC343331 & KC343573 \\
\hline D. eres & CBS 439.82; BBA P-407; IMI 162181a T & KC343090 & KC343816 & KC344058 & KC343332 & KC343574 \\
\hline D. eugeniae & CBS 444.82 & KC343098 & KC343824 & KC344066 & KC343340 & KC343582 \\
\hline D. fibrosa & CBS 109751; AR 3425 & KC343099 & KC343825 & KC344067 & KC343341 & KC343583 \\
\hline D. fibrosa & CBS 113830; UPSC 2117 & KC343100 & KC343826 & KC344068 & KC343342 & KC343584 \\
\hline D. foeniculacea & CBS 123208; Di-C004/5 T & KC343104 & KC343830 & KC344072 & KC343346 & KC343588 \\
\hline D. foeniculacea & CBS 123209; Di-C004/4 T & KC343105 & KC343831 & KC344073 & KC343347 & КC343589 \\
\hline D. foeniculacea & CBS $187.27 \mathrm{~T}$ & KC343107 & KC343833 & KC344075 & KC343349 & KC343591 \\
\hline D. ganjae & CBS 180.91; ILLS $43621 \mathrm{~T}$ & KC343112 & KC343838 & KC344080 & KC343354 & KC343596 \\
\hline D. gardeniae & CBS 288.56 & KC343113 & KC343839 & KC344081 & KC343355 & KC343597 \\
\hline & & & & & (Continued & next page) \\
\hline
\end{tabular}

\footnotetext{
a Sequence names are the currently accepted species name determined by Gomes et al. (2013). Extype cultures are denoted by the letter T.

${ }^{\mathrm{b}}$ ITS $=$ internal transcribed spacer, EF1 $\alpha=$ translation elongation factor $1-\alpha$, TUB $=\beta$-tubulin, $\mathrm{CAL}=$ calmodulin, and HIS $=$ histone.
} 
Table 3. (Continued from previous page)

\begin{tabular}{|c|c|c|c|c|c|c|}
\hline \multirow[b]{2}{*}{ Sequence name } & \multirow[b]{2}{*}{ Isolate } & \multicolumn{5}{|c|}{ GenBank accession numbers ${ }^{b}$} \\
\hline & & ITS & EF1 $\alpha$ & TUB & CAL & HIS \\
\hline D. helianthi & CBS 344.94 & KC343114 & KC343840 & KC344082 & KC343356 & KC343598 \\
\hline D. helianthi & CBS $592.81 \mathrm{~T}$ & KC343115 & KC343841 & KC344083 & KC343357 & KC343599 \\
\hline D. cf. heveae 1 & CBS 852.97 & KC343116 & KC343842 & KC344084 & KC343358 & KC343600 \\
\hline D. cf. heveae 2 & CBS 681.84 & KC343117 & KC343843 & KC344085 & KC343359 & KC343601 \\
\hline D. hickoriae & CBS $145.26 \mathrm{~T}$ & KC343118 & KC343844 & KC344086 & KC343360 & KC343602 \\
\hline D. hongkongensis & CBS 115448; HKUCC 9104; AT 646 DF 24 T & KC343119 & KC343845 & KC344087 & KC343361 & KC343603 \\
\hline D. hordei & CBS 481.92 & KC343120 & KC343846 & KC344088 & KC343362 & KC343604 \\
\hline D. impulsa & CBS 114434; UPSC 3052 & KC343121 & KC343847 & KC344089 & KC343363 & KC343605 \\
\hline D. impulsa & CBS 141.27 & KC343122 & KC343848 & KC344090 & KC343364 & KC343606 \\
\hline D. inconspicua & CBS 133813; LGMF930; CPC 20306 T & KC343123 & KC343849 & KC344091 & KC343365 & KC343607 \\
\hline D. inconspicua & LGMF922; CPC 20298 & KC343124 & KC343850 & KC344092 & KC343366 & KC343608 \\
\hline D. inconspicua & LGMF931; CPC 20307 & KC343125 & KC343851 & KC344093 & KC343367 & KC343609 \\
\hline D. infecunda & CBS 133812; LGMF906; CPC 20282 T & KC343126 & KC343852 & KC344094 & KC343368 & $\mathrm{KC} 343610$ \\
\hline D. infecunda & LGMF908; CPC 20284 & KC343127 & KC343853 & KC344095 & KC343369 & KC343611 \\
\hline D. infecunda & LGMF912; CPC 20288 & KC343128 & KC343854 & KC344096 & KC343370 & KC343612 \\
\hline D. juglandina & CBS 121004; DP 0659 & KC343134 & KC343860 & KC344102 & KC343376 & KC343618 \\
\hline D. longispora & CBS $194.36 \mathrm{~T}$ & KC343135 & KC343861 & KC344103 & KC343377 & KC343619 \\
\hline D. lusitanicae & CBS 123212; Di-C001/5 T & KC343136 & KC343862 & KC344104 & KC343378 & KC343620 \\
\hline D. lusitanicae & CBS 123213; Di-C001/3 & KC343137 & KC343863 & KC344105 & KC343379 & KC343621 \\
\hline D. manihotia & CBS 505.76 & KC343138 & KC343864 & KC344106 & KC343380 & $\mathrm{KC} 343622$ \\
\hline D. mayteni & CBS 133185; LGMF938; CPC 20314 T & KC343139 & KC343865 & KC344107 & KC343381 & KC343623 \\
\hline D. megalospora & CBS 143.27 & KC343140 & KC343866 & KC344108 & KC343382 & KC343624 \\
\hline D. melonis & CBS 435.87 & KC343141 & KC343867 & KC344109 & KC343383 & KC343625 \\
\hline D. melonis & CBS $507.78 \mathrm{~T}$ & KC343142 & KC343868 & KC344110 & KC343384 & KC343626 \\
\hline D. musigena & CBS 129519; CPC 17026 T & KC343143 & KC343869 & KC344111 & KC343385 & KC343627 \\
\hline D. neilliae & CBS 144.27 & KC343144 & KC343870 & $\mathrm{KC} 344112$ & KC343386 & $\mathrm{KC} 343628$ \\
\hline D. neoarctii & CBS 109490; GB 6421; AR 3450 T & KC343145 & KC343871 & KC344113 & KC343387 & KC343629 \\
\hline D. nobilis & CBS 113470; DAOM 226800 & KC343146 & KC343872 & KC344114 & KC343388 & KC343630 \\
\hline D. nobilis & CBS 116953; NZ-26 & KC343147 & KC343873 & KC 344115 & KC343389 & KC343631 \\
\hline D. nobilis & CBS 116954; NZ-27 & KC343148 & KC343874 & KC344116 & KC343390 & KC343632 \\
\hline D. nomurai & CBS 157.29 & KC343154 & KC343880 & KC344122 & KC343396 & KC343638 \\
\hline D. novem & CBS $127269 ; 5-27 / 3-1$ & KC343155 & KC343881 & KC344123 & KC343397 & KC343639 \\
\hline D. novem & CBS $127270 ; 4-27 / 3-1 \mathrm{~T}$ & KC343156 & KC343882 & KC344124 & KC343398 & KC343640 \\
\hline D. novem & CBS $127271 ; 5 / 27 / 3-3$ & KC343157 & KC343883 & KC344125 & KC343399 & KC343641 \\
\hline D. oncostoma & CBS 100454 & KC343160 & KC343886 & KC344128 & KC343402 & KC343644 \\
\hline D. oncostoma & CBS 589.78 & KC343162 & KC343888 & KC344130 & KC343404 & KC343646 \\
\hline D. oncostoma & CBS 809.85 & KC343163 & KC343889 & KC344131 & KC343405 & KC343647 \\
\hline D. oxe & CBS 133186; LGMF942; CPC 20318 T & KC343164 & KC343890 & KC344132 & KC343406 & KC343648 \\
\hline D. oxe & CBS 133187; LGMF936; CPC 20312 & KC343165 & KC343891 & KC 344133 & KC343407 & KC343649 \\
\hline D. oxe & LGMF915; CPC 20291 & KC343166 & KC343892 & KC344134 & KC343408 & KC343650 \\
\hline D. padi var. padi & CBS 114200; UPSC 2569 & KC343169 & KC343895 & KC344137 & KC343411 & KC343653 \\
\hline D. padi var. padi & CBS 114649; UPSC 3496 & KC 343170 & KC343896 & KC 344138 & KC343412 & KC343654 \\
\hline D. paranensis & CBS 133184; LGMF929; CPC 20305 T & KC343171 & KC343897 & KC344139 & KC343413 & KC343655 \\
\hline D. perjuncta & CBS 109745; ARSEF 3461; AR 3461 T & KC343172 & KC343898 & KC344140 & KC343414 & KC343656 \\
\hline D. perseae & CBS 151.73 & KC343173 & KC343899 & KC344141 & KC343415 & KC343657 \\
\hline D. phaseolorum & CBS 113425 & KC343174 & KC343900 & KC344142 & KC343416 & KC343658 \\
\hline D. phaseolorum & CBS 116019; STAM 30 & KC343175 & KC343901 & KC344143 & KC343417 & KC343659 \\
\hline D. phaseolorum & CBS 116020; STAM 31 & KC343176 & KC343902 & KC344144 & KC343418 & KC343660 \\
\hline D. pseudomangiferae & CBS $101339 \mathrm{~T}$ & KC343181 & KC343907 & KC344149 & KC343423 & KC343665 \\
\hline D. pseudomangiferae & CBS 388.89 & KC343182 & KC343908 & KC 344150 & KC343424 & KC343666 \\
\hline D. pseudophoenicicola & CBS 176.77 & KC343183 & KC343909 & KC344151 & KC343425 & KC343667 \\
\hline D. pseudophoenicicola & CBS $462.69 \mathrm{~T}$ & KC343184 & KC343910 & KC344152 & KC343426 & KC343668 \\
\hline D. pustulata & CBS 109742; AR 3430 & KC343185 & KC343911 & KC344153 & KC343427 & KC343669 \\
\hline D. pustulata & CBS 109760; AR 3535 & KC343186 & KC343912 & KC344154 & KC343428 & KC343670 \\
\hline D. pustulata & CBS 109784; AR 3419 & KC343187 & KC343913 & KC344155 & KC343429 & KC343671 \\
\hline D. raonikayaporum & CBS 133182; LGMF923; CPC 20299 T & KC343188 & KC343914 & KC344156 & KC343430 & KC343672 \\
\hline D. rhoina & CBS 146.27 & KC343189 & KC343915 & KC344157 & KC343431 & KC343673 \\
\hline D. saccarata & CBS 116311; STE-U 3743; CPC 3743 T & KC343190 & KC343916 & KC344158 & KC343432 & KC343674 \\
\hline D. schini & CBS 133181; LGMF921; CPC 20297 T & KC343191 & KC343917 & KC344159 & KC343433 & KC343675 \\
\hline D. schini & LGMF910; CPC 20286 & KC343192 & KC343918 & KC 344160 & KC343434 & KC343676 \\
\hline D. sclerotioides & CBS 296.67; ATCC 18585; IMI 151828 T & KC343193 & KC343919 & KC344161 & KC343435 & KC343677 \\
\hline D. sclerotioides & CBS 710.76; PD 76/674 & KC343194 & KC343920 & KC344162 & KC343436 & KC343678 \\
\hline D. scobina & CBS 251.38 & KC343195 & KC343921 & KC344163 & KC343437 & KC343679 \\
\hline D. sojae & CBS 100.87 & KC343196 & KC343922 & KC344164 & KC 343438 & KC343680 \\
\hline D. sojae & CBS 116023; STAM 35 & KC343198 & KC343924 & KC344166 & KC343440 & KC343682 \\
\hline
\end{tabular}


Table 3. (Continued from previous page)

\begin{tabular}{|c|c|c|c|c|c|c|}
\hline \multirow[b]{2}{*}{ Sequence name } & \multirow[b]{2}{*}{ Isolate } & \multicolumn{5}{|c|}{ GenBank accession numbers ${ }^{b}$} \\
\hline & & ITS & EF1 $\alpha$ & TUB & CAL & HIS \\
\hline D. sojae & CBS 659.78; NRRL 13656 & KC343201 & KC343927 & KC344169 & KC343443 & KC343685 \\
\hline Diaporthe sp. 1 & CBS 119639; B 11861 & KC343202 & KC343928 & KC344170 & KC343444 & KC343686 \\
\hline Diaporthe sp. 2 & LGMF947; CPC 20323 & KC343203 & KC343929 & KC344171 & KC343445 & KC343687 \\
\hline Diaporthe sp. 2 & LGMF932; CPC 20308 & KC343204 & KC343930 & $\mathrm{KC} 344172$ & KC343446 & KC343688 \\
\hline Diaporthe sp. 3 & CBS 287.29 & KC343205 & KC343931 & KC344173 & KC343447 & KC343689 \\
\hline Diaporthe sp. 5 & CBS 125575 & KC343207 & KC343933 & KC344175 & KC343449 & KC343691 \\
\hline Diaporthe sp. 6 & CBS 115584; HKUCC 7784; AT 7 & KC343208 & KC343934 & KC344176 & KC 343450 & KC343692 \\
\hline Diaporthe sp. 7 & CBS 115595; HKUCC 10129 & KC343209 & KC343935 & KC344177 & KC343451 & KC343693 \\
\hline Diaporthe sp. 7 & CBS 458.78 & KC343210 & KC343936 & KC344178 & KC343452 & KC343694 \\
\hline Diaporthe sp. 8 & LGMF925; CPC 20301 & KC343211 & KC343937 & KC344179 & KC343453 & KC343695 \\
\hline D. stictica & CBS 370.54 & KC343212 & KC343938 & KC344180 & KC343454 & KC343696 \\
\hline D. subordinaria & CBS 101711 & KC343213 & KC343939 & KC344181 & KC343455 & KC343697 \\
\hline D. subordinaria & CBS 464.90 & KC343214 & KC343940 & KC344182 & KC343456 & KC343698 \\
\hline D. tecomae & CBS 100547 & KC343215 & KC343941 & KC344183 & KC343457 & KC343699 \\
\hline D. terebinthifolii & CBS 133180; LGMF914; CPC 20290 T & KC343216 & KC343942 & KC344184 & KC343458 & KC343700 \\
\hline D. terebinthifolii & LGMF907; CPC 20283 & KC343217 & KC343943 & KC344185 & KC343459 & KC343701 \\
\hline D. terebinthifolii & LGMF909; CPC 20285 & KC343218 & KC343944 & KC344186 & KC343460 & KC343702 \\
\hline D. toxica & CBS 534.93; ATCC $96741 \mathrm{~T}$ & KC 343220 & KC343946 & KC344188 & KC343462 & KC343704 \\
\hline D. toxica & CBS 535.93 & KC343221 & KC343947 & KC344189 & KC343463 & KC343705 \\
\hline D. toxica & CBS 546.93 & KC343222 & KC343948 & KC344190 & KC343464 & KC343706 \\
\hline D. vaccinii & CBS 122115; FAU 590 & KC343226 & KC343952 & KC344194 & KC343468 & KC343710 \\
\hline D. vaccinii & CBS 122116; DF 5022 & KC343227 & KC343953 & KC344195 & KC343469 & KC343711 \\
\hline D. vaccinii & CBS 160.32; IFO $32646 \mathrm{~T}$ & KC343228 & KC343954 & KC344196 & KC343470 & KC343712 \\
\hline D. vexans & CBS 127.14 & KC343229 & KC343955 & KC344197 & KC343471 & KC343713 \\
\hline D. viticola & CBS 113201; STE-U 5683; CPC 5683 T & KC343234 & KC343960 & KC344202 & KC343476 & KC343718 \\
\hline D. viticola & CBS 759.95 & KC343242 & KC343968 & KC 344210 & KC343484 & KC343726 \\
\hline D. viticola & CBS 794.96 & KC343243 & KC343969 & KC344211 & KC343485 & KC343727 \\
\hline D. woodii & CBS 558.93 & KC343244 & KC343970 & KC344212 & KC343486 & KC 343728 \\
\hline D. woolworthii & CBS 148.27 & KC343245 & KC343971 & KC 344213 & KC343487 & KC343729 \\
\hline Diaporthella corylina & CBS 121124; AR 4131 & KC343004 & KC343730 & KC343972 & KC343246 & KC343488 \\
\hline
\end{tabular}

(micrograms per milliliter) for testing based on label rates for Phoma and Phomopsis control for trifloxystrobin and pyraclostrobin and boscalid mix (Table 4). For pyraclostrobin, we calculated rates that would be equal to the concentration of pyraclostrobin in the mixed fungicide (Table 4). Fungicides were added to 1/2PDA agar to obtain the desired concentrations in micrograms per milliliter. D. humulicola cultures were grown on 1/2PDA from the CT2018-1 and CT2018-3 isolates. Plugs taken from the $D$. humulicola cultures with a 5-mm core borer were placed in the center of the fungicide-amended plates in replicates of four and onto four unamended (untreated control) plates. Cultures were grown at $22^{\circ} \mathrm{C}$ in complete darkness for $96 \mathrm{~h}$. The diameter of the cultures was measured at two perpendicular angles. Relative mycelial growth (RMG) was calculated as growth of fungicide-amended culture divided by growth on unamended (untreated control) media. The experiment was repeated twice for trifloxystrobin at the original calculated concentrations. For pyraclostrobin and boscalid and pyraclostrobin alone, concentrations were reduced due to complete growth inhibition at all original concentrations and subsequently repeated twice (Table 4).

\section{Results}

Field conditions. The growing season in 2018 had similar air temperatures at both locations (Table 5). When symptoms appeared in July at Windsor, the average temperature was $24.4^{\circ} \mathrm{C}$, with $23.9^{\circ} \mathrm{C}$ in August at both Windsor and Hamden. Average monthly rainfall was greater in July and August compared with May and June at both locations, coinciding with the appearance of symptoms (Table 5). In 2019 , leaf spots were observed following wet and warm weather conditions at both locations.

Symptoms in the field. Symptoms occurred from the spring to summer and appeared to be small dark spots on the leaves, often water soaked in the early stage, and expanded into ellipsoid, brownishgray lesions, often with white rings, or with chlorotic margins. In late summer, dark-brown to black pycnidia developed in the lesions on the adaxial side under moist conditions. Milky-colored conidial masses oozed out from the ostioles under humid or wet conditions. Leaf spot lesions were not delimited by veins and often associated with leaf margins (Fig. 1). As disease progressed, cones became infected, showing dark reddish-brown margins of bracts (Fig. 1C).

Koch's postulates test. Inoculated plants displayed leaf lesions with brownish-gray lesions with white concentric rings, whereas the untreated controls did not display symptoms. Cultures resulting from isolations from inoculated plant leaf lesions had identical appearance to $D$. humulicola on 1/2PDA and microscopic examination found identical conidia.

Phylogenetic analyses. All three isolates had identical ITS sequences. BLAST analysis of the ITS region placed the CT2018-1 isolate as a member of the genus Diaporthe, with the closest GenBank accession being an unidentified Diaporthe sp. isolate CLJ-1 (accession number LC373144) isolated from Cinchona ledgeriana L. at $96.55 \%$ identity. For the EF1 $\alpha$ locus, the three isolates shared identical sequences with the closest BLAST match as Diaporthe anacardii (Early \& Punith.) R. R. Gomes, C. Glienke, \& Crous, accession number MK442692, at $81.44 \%$ identity. For TUB, sequences from all three isolates had identical sequences and the nearest BLAST hit was an unidentified Phomopsis sp. strain Pho08 (accession number HQ586907) isolated from Vitis vinifera L. at $95.75 \%$ identity. All three isolates shared identical HIS locus sequences, with the closest BLAST match being $D$. eres Nitschke, accession number MG516978. At the CAL locus, all three isolates presented with sequence variation. Pairwise distances were $0.63 \%$ for CT2018-1 and CT2018-2, $0.16 \%$ for CT2018-1 and CT2018-3, and 0.16\% for CT2018-2 and CT2018-3. The closest match revealed by a BLAST search was D. perjuncta Niessl., accession number KC343414, at $83.2 \%$ identity. The $28 \mathrm{~S}$ locus sequence was identical for all three isolates and the closest BLAST match was Stenocarpella maydis 
(Berk.) B. Sutton, accession number KP164561, at $99.06 \%$ identity. The three isolates were not identical for the $r p b 2$ gene sequences, with pairwise distances of $1.4 \%$ between CT2018-1 and CT20182, $0.87 \%$ for CT2018-1 and CT2018-3, and 0.52\% for CT2018-2 and CT2018-3. A BLAST search of CT2018-1 was most similar to Phomopsis viticola (Sacc.) Sacc. isolate PhoCT2L (currently accepted name: D. ampelina), accession number HQ446836, isolated from a Vitis sp. at $92.73 \%$ sequence identity.

A maximum-likelihood phylogenetic tree of Phoma spp. and Diaporthaceae species placed D. humulicola isolates CT2018-1, CT2018-2, and CT2018-3 within Diaporthe, Diaporthaceae, Diparothales and separated out the Phoma spp. and Didymella macrostoma (Mont.) Qian Chen \& L. Cai with 100\% bootstrap support (Fig. 3).

Maximum-likelihood and Bayesian analyses showed that $D$. humulicola clearly separated from the outgroup, Diaporthella corylina Lar. N. Vassiljeva, as well as Diaporthe acaciigena Crous, Pascoe \& Jacq. Edwards, D. pustulata Sacc., D. amygdali (Delacr.) Udayanga, Crous \& K. D. Hyde, D. australafricana Crous \& Van Niekerk, D. viticola Nitschke, D. cynaroidis Marinc. M. J. Wingf. $\&$ Crous, D. beckhausii Nitschke, D. heveae Petch, and D. toxica P. M. Will., Highet, W. Gams \& Sivasith. (Fig. 4; Supplementary Fig. S1). All three isolates of $D$. humulicola grouped together with $100 \%$ significance (Fig. 4). D. humulicola clustered most closely (98.4\%) with D. ambigua, D. longispora, D. scerlotiodies, D. mayteni, D. raonikayaporum, Diaporthe. sp. 2, D. angelica, D. subordinaria, D. arctii, D. neoarctii, D. cuppatea, D. lusitanicae, D. novem, $D$. infecunda, D. batatas, D. citri, D. sojae, Diaporthe sp. $1, D$. convolvuli, D. endophytica, D. phaseolorum, D. melonis, D. helianthin, D. hordei, D. vexans, D. megalospora, D. schini, D. tecomae, D. terebinthifolii, D. ganjae, D. manihota, D. oxe, Diaporthe sp. 3, D. paranensis, D. brasiliensis, and Diaporthe sp. 5 (Fig. 4).

Taxonomy. The results of the molecular analysis and observations of morphological characteristics in planta and in culture support the conclusion that all three isolates-CT2018-1, CT2018-2, and CT2018-3 - are a single, new species of Diaporthe. Two subcultures of CT2018-3 produced rhizomorph-like sclerotia in which a number of pycnidia were embedded with sporulation structures similar to the pycnidia that were produced in cell culture and on leaf tissue (Fig. 2g). The rhizomorph structures were observed after $51 / 2$ months of growth on 1/2PDA at ambient room temperature.

Diaporthe humulicola E. B. Allan-Perkins, D. W. Li, N. P. Schultes \& J. A. LaMondia sp. nov. MycoBank number MB832379. Sexual state: undetermined. Conidiomata: pycnidial, solitary or aggregated, conical to globose or flask-like, brown to dark brown, up to $267 \mu \mathrm{m}$ diam. on leaves and $1,250 \mu \mathrm{m}$ on 1/2PDA, subepidermal in leaf and petiole tissues, erumpent through surface, unilocular (Fig. 2b). Ostiole: present. Wall: parenchymatous, textura angularis. Conidiophores: reduced to conidiogenous cells, hyaline, unbranched, not septate, growing along all walls of pycnidium, (17.2) 21.7-26.6 (29.5) × (1.2) 1.4-2.7 (3.7) $\mu \mathrm{m}$ (mean \pm standard deviation [SD]: $24.7 \pm 2.9 \times 2 \pm 0.6, n=30$ ) (Fig. 2d). Conidiogenous cells: enteroblastic, monophialidic, determinate, unbranched, hyaline, smooth, tubulate, cylindrical, (2.5) 3.4-6.4 (7.4) $\times(1.2)$ 2.0-3.3 (4.9) $\mu \mathrm{m}$ (mean \pm SD: $4.9 \pm 1.5 \times 2.6 \pm 0.7, n=30$ ) (Fig. 2d). $\alpha$ Conidia: hyaline, eguttulate, rarely 1 to multiguttulate, 1celled, smooth, cylindrical or clavate with obtuse ends, some constricted in the middle, occasionally becoming dumb-bell shaped, (3) 4.4-11.6 (15) $\times(2.5)$ 2.7-5.4 (7.5) $\mu \mathrm{m}$ (mean \pm SD: $8 \pm 3.6 \times$ $4.1 \pm 1.3, n=30$ ) (Fig. $2 \mathrm{e}$ and f). $\beta$ and $\gamma$ Conidia: not observed.

Culture characteristics: Colony grown on 1/2PDA. Irregular form, flat elevation, undulate margin, white and brown-gray bands, pycnidia sparse and irregularly dispersed over agar surface (Fig. 2a).

Holotype: U.S.A., Connecticut, Windsor, $41^{\circ} 51^{\prime} 0.65^{\prime \prime} \mathrm{N}, 72^{\circ} 39^{\prime}$ $38.16^{\prime \prime} \mathrm{W}$, Humulus lupulus cultivar AlphAroma leaf, 31 July 2018, E. B. Allan-Perkins, UAMH 12076 (= CT2018-1). Holotype specimen is a living specimen being maintained via lyophilization at UAMH Centre for Global Microfungal Biodiversity, The Gage Research Institute, University of Toronto, Toronto, Canada.

Etymology: Latin; Humulus referring to host genus, Humulus, and -cola, ones that grow on.

Additional materials examined: USA, Connecticut, Windsor, $41^{\circ} 51^{\prime} 0.65^{\prime \prime} \mathrm{N}, 72^{\circ} 39^{\prime} 38.16^{\prime \prime} \mathrm{W}$, Humulus lupulus cultivar Saaz cone, 31 July 2018, E. B. Allan-Perkins, (CT2018-2). U.S.A., Connecticut, Hamden, $41^{\circ} 24^{\prime} 20.34^{\prime \prime} \mathrm{N}, 72^{\circ} 54^{\prime} 26.78^{\prime \prime} \mathrm{W}$, Humulus lupulus cultivar Newport, 7 August 2018, M. Salvas, (CT2018-3).

Host/distribution: from Humulus lupulus in Windsor and Hamden, Connecticut, U.S.A.

Comments: In addition to the holotype described above, conidiophores, conidiogenous cells, and conidia were measured from two additional isolates. For isolate 2018-3 (isolated from leaf of cultivar Newport collected in Hamden, CT), conidiophores measured (14.8) 17.7-24.5 (29.5) × (1.8) 1.9-2.7 (3.1) $\mu \mathrm{m}$ (mean \pm SD: $21.1 \pm 3.4 \times$ $2.3 \pm 0.4, n=30$ ), conidiogenous cells measured (4.9) 7.4-12.1 $(16.0) \times(1.8) 2.1-3.7(6.2) \mu \mathrm{m}($ mean \pm SD: $9.7 \pm 2.4 \times 2.9 \pm 0.8$, $n=30)$, and $\alpha$ conidia measured (6.2) 7.3-10.2 (12.3) $\times(2.5)$ 2.5-4.4 (5.4) $\mu \mathrm{m}$ (mean \pm SD: $8.8 \pm 1.5 \times 3.4 \pm 0.9, n=30$ ). For isolate 2018-9 (isolated from leaf of cultivar AlphAroma collected in Hamden, CT), conidiophores measured (11.1) 14.4-22.4 (24.6) $\times$ (1.8) $1.8-3.1(4.9) \mu \mathrm{m}($ mean \pm SD: $18.4 \pm 4.0 \times 2.5 \pm 0.7, n=$ 30), conidiogenous cells measured (2.5) 5.9-11.1 (14.8) $\times(1.8)$ $2.2-3.9(6.2) \mu \mathrm{m}$ (mean \pm SD: $8.5 \pm 2.6 \times 3.0 \pm 0.8, n=30)$, and $\alpha$ conidia measured (6.9) 7.8-10.6 (12.3) $\times(2.5) 3.0-4.9(6.2) \mu \mathrm{m}$ (mean \pm SD: $9.2 \pm 1.4 \times 3.9 \pm 1.0, n=30$ ). According to both morphological characteristics and phylogenetic analyses (Fig. 3), all three isolates belong to $D$. humulicola.

D. humulicola shows similar symptoms on common hop as Phoma exigua and $P$. aliena but its morphology lacks scleroplectenchyma in pycnidia and molecular analysis shows that it is a member of the genus Diaporthe.

Table 5. Weather conditions in Windsor and Hamden, CT hop yards during Diaporthe humulicola infection of hop in 2018

\begin{tabular}{lccccc}
\hline & \multicolumn{2}{c}{ Temperature $\left({ }^{\circ} \mathbf{C}\right)$} & & \multicolumn{2}{c}{ Monthly rainfall $(\mathbf{c m})$} \\
\cline { 2 - 3 } Month & Windsor & Hamden & & Windsor & Hamden \\
\hline March & 3.09 & 3.33 & & 16.52 & 16.65 \\
April & 6.84 & 6.99 & & 37.55 & 44.90 \\
May & 17.96 & 17.03 & & 15.29 & 22.06 \\
June & 20.17 & 19.66 & & 22.58 & 26.32 \\
July & 24.36 & 23.74 & & 30.26 & 29.94 \\
August & 23.89 & 23.90 & & 42.39 & 25.87 \\
September & 19.08 & 19.36 & & 48.45 & 65.03 \\
\hline
\end{tabular}

Table 4. Fungicide concentrations used for in vitro analysis of Diaporthe humulicola fungicide sensitivity

\begin{tabular}{lccc}
\hline Parameters & Trifloxystrobin (Flint) & Pyraclostrobin + Boscalid (Pageant) & Pyraclostrobin (Insignia) $^{\text {a }}$ \\
\hline Recommended rate & $1 \mathrm{oz} / 15-30 \mathrm{gal}$ & $8-12 \mathrm{oz} / 100 \mathrm{gal}$ & $\mathrm{NA}$ \\
Percent active ingredient & 50.0 & $12.8^{\mathrm{b}}$ & 20.0 \\
Active ingredient $(\mu \mathrm{g} / \mathrm{ml})$ & 249.67 & $76.70-115.05$ & $\mathrm{NA}$ \\
Concentrations tested $(\mu \mathrm{g} / \mathrm{ml})$ & $10,100,250$ & $40,80,120$ & $25,50,75$ \\
Concentrations tested in repeated experiments & $\mathrm{NA}$ & $20,10,1$ & $12,6,0.6$ \\
$(\mu \mathrm{g} / \mathrm{ml})$ & & & \\
\hline
\end{tabular}

\footnotetext{
${ }^{\text {a }}$ Calculated concentrations to be tested to equal pyraclostrobin in micrograms per milliliter in Pageant. NA = not available.
}

${ }^{b}$ Percent active ingredient for pyraclostrobin only. 
Anamorphic states of some taxa of Diaporthe are trimorphic and develop $\alpha, \beta$, and $\gamma$ conidia (Chi et al. 2007). A number of species of Diaporthe (Phomopsis) develop only $\alpha$ conidia (Chi et al. 2007; Gomes et al. 2013; Sutton 1980; Yang et al. 2018b). Among the species with only $\alpha$ conidia, the ones similar to D. humulicola with unbranched conidiophores include D. acericola Dissan., Camporesi \& K. D. Hyde, D. alangii C. M. Tian \& Qin Yang, D. alleghaniensis R. H. Arnold, D. bicincta (Cooke \& Peck) Sacc., D. bohemiae Guarnaccia, Eichmeier \& Crous, D. brasiliensis R. R. Gomes, Glienke \& Crous, D. celastrina Ellis \& Barthol., D. cichorii Dissan., Camporesi

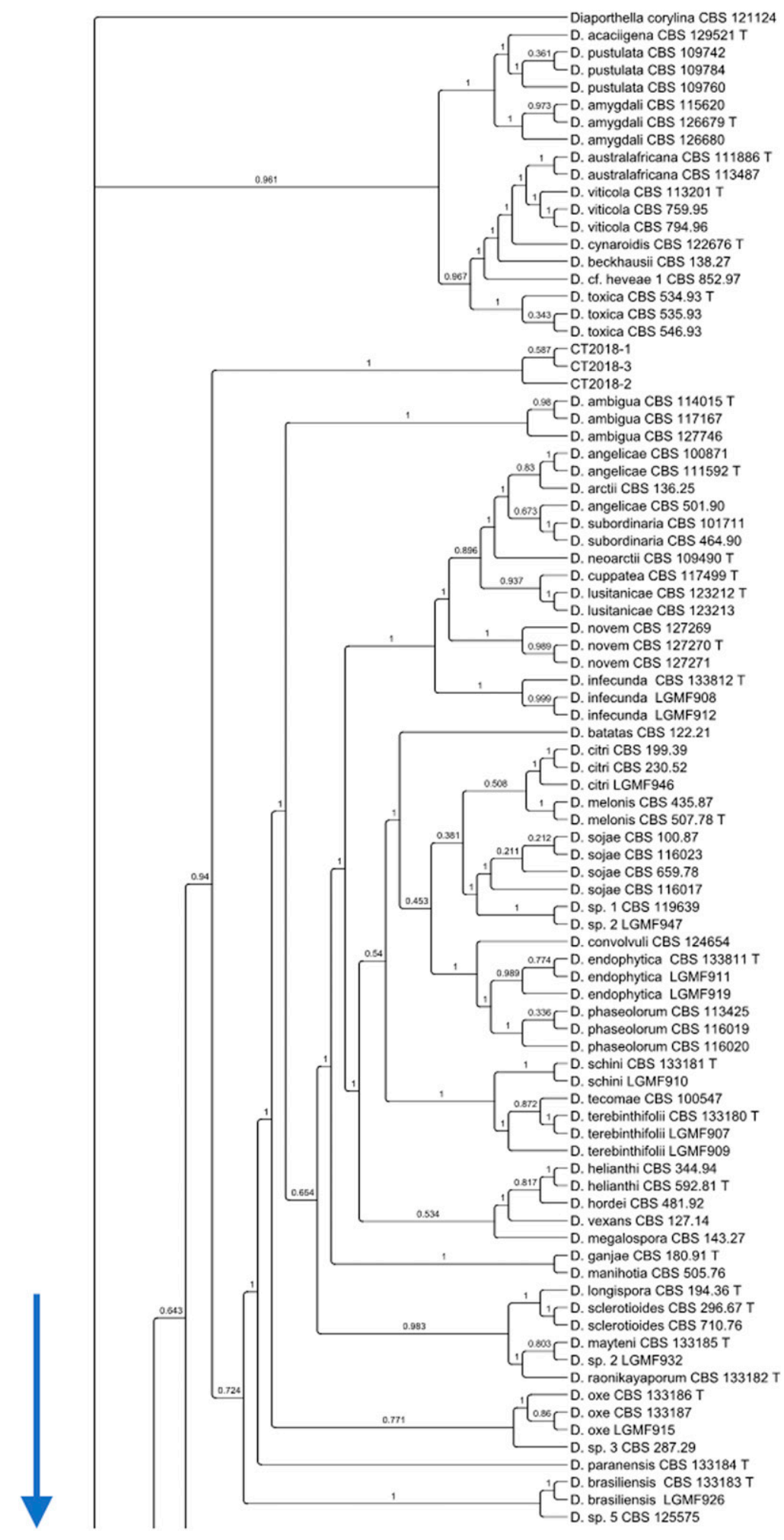

(Continued on next page)

Fig. 4. Phylogenetic tree of Diaporthe humulicola isolates CT2018-1, CT2018-2, and CT2018-3 with members of Diaporthales based on Bayesian inference analyzed for the combined dataset of internal transcribed spacer, translation elongation factor $\alpha-1, \beta$-tubulin, histone, and calmodulin sequences. Tree sampling frequency was 1,000 generations. Branches that received Bayesian posterior probabilities of 0.95 were set as significantly supported. Diaporthella corylina was included as the outgroup. 
\& K. D. Hyde, D. conica C. M. Tian \& Qin Yang, D. dorycnii Dissan., Camporesi \& K. D. Hyde, D. eres, D. helicis Niessl, D. hungariae Guarnaccia, Armengol \& K. Z. Váczy, D. kadsurae C. M. Tian \& Qin Yang, D. longicolla (Hobbs) J. M. Santos, Vrandečić \& A. J. L. Phillips, D. melonis Beraha \& M. J. O'Brien, D. pulla Nitschke, and D. sojae Lehman, (Chi et al. 2007; Dissanayake et al. 2017; Gomes et al. 2013; Guarnaccia et al. 2018; Sutton 1980; Udayanga et al. 2015; Yang et al. 2018b). The conidial shape, size, and guttation will differentiate these species from $D$. humulicola (cylindrical or clavate, some constricted in the middle, occasionally becoming dumb-bell shaped, $4.0-12.2 \times 2.2-5.0 \mu \mathrm{m})$. Phylogenetic relationships further showed that these taxa were different from D. humulicola (Fig. 4).

Several species such as Phomopsis abdita (Sacc.) Traverso, P. lantanae (M. E. A. Costa \& Sousa da Câmara) B. Sutton, and P. terminaliae (Henn.) B. Sutton share some morphological characteristics with D. humulicola (Chi et al. 2007; Sutton 1980) but do not have molecular data available for phylogenetic analysis. $P$. terminaliae has large $\alpha$ conidia $(10-15 \times 3-4 \mu \mathrm{m})$ to separate it from $D$.

Fig. 4. (Continued from previous page)

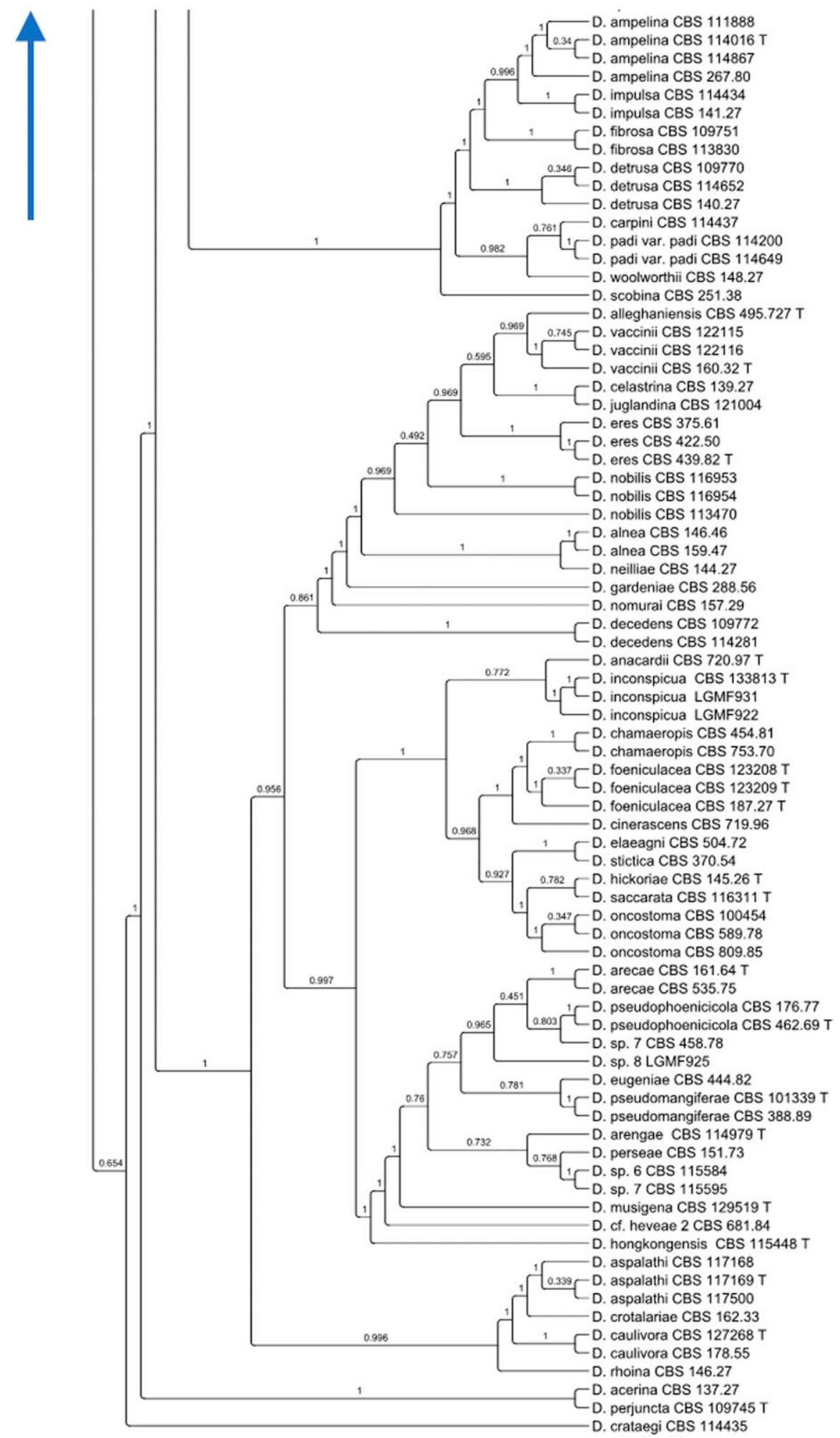


humulicola. P. abdita has $\alpha$ conidia fusiform to ellipsoid, 2-4 guttulate, $8-10 \times 2 \mu \mathrm{m}$ and $P$. lantanae, fusiform, biguttulate, $6.5-9 \times$ $2.5 \mu \mathrm{m}$, which will differentiate the two from $D$. humulicola by the shape and guttation.

$D$. humulicola is distinct from its closest relative from phylogenetic analysis, D. brasilensis, based on the latter having septate conidiophores and conidia being generally smaller than those of $D$. humulicola and having a rounded apex compared with the obtuse ends of $D$. humulicola conidia.

Fungicide efficacy. All three fungicides reduced fungal growth in vitro compared with unamended plates (Figs. 5 and 6). Trifloxystrobin had the lowest inhibition of $D$. humulicola growth, with average RMG of 54\% for CT2018-1 and 23\% at $10 \mu \mathrm{g} / \mathrm{ml}$ (Fig. 5). Pyraclostrobin combined with boscalid inhibited all growth at concentrations at and above $10 \mu \mathrm{g} / \mathrm{ml}$ (Fig. 6). At $1 \mu \mathrm{g} / \mathrm{ml}$, CT2018-3 was inhibited completely but CT2018-1 had an average RMG of $2.73 \%$ (Fig. 6). Growth of CT2018-3 was completely inhibited with pyraclostrobin alone at $12 \mu \mathrm{g} / \mathrm{ml}$ (Fig. 7). For pyraclostrobin at $0.6 \mu \mathrm{g} / \mathrm{ml}$, CT2018-1 had an average RMG of 4.99\% and CT2018-3 had an average RMG of $2.04 \%$ (Fig. 7). CT2018-1 tended to grow better under all fungicide concentrations compared with CT2018-3 (Figs. 5, 6, and 7).

\section{Discussion}

We have demonstrated the presence of a new pathogen, D. humulicola, on common hop in Connecticut discovered in field plots in both 2018 and 2019. The pathogen results in symptoms similar to Phoma wilt on hop, including similar leaf lesions and browning of cones (Mahaffee et al. 2009). Phoma spp. have been implicated in causing minor infections of hop plants in the United States and as a more destructive pathogen in Europe, New Zealand, and China. More recently, Phoma spp. have been implicated in causing loss of hop cones due to browning late in the season in Vermont (Darby 2017). However, both morphological and phylogenetical analyses revealed that $D$. humulicola is distinct from Phoma spp. The latter are characterized by pycnidia that contain scleroplectenchyma, which was not observed for $D$. humulicola. Phylogenetically, Phoma belongs to Pleosporales, Dothideomycetes, while Diaporthe is placed within Diaporthales, Sordariomycetes (MycoBank 2019). Of the species observed on hop, $P$. exigua, $P$. aliena, $P$. herbarium, and $P$. macrostoma differ in morphology based on the lack of scleroplectenchyma in D. humulicola and that the latter has much larger conidia than those of the three Phoma spp. Molecular analysis based on NCBI Blast results and phylogenetic analyses clearly demonstrate that the new pathogen on hop is a member of the genus Diaporthe.

Although 28S blast results found the closest match to be $S$. maydis, we do not believe that $D$. humulicola is a member of this genus. $S$. maydis has pigmented $\alpha$ conidia and is mostly restricted to maize (Crous et al. 2006; Gao et al. 2017; Lamprecht et al. 2011). Phylogenetically, it is placed within the family Diaporthaceae. However, $D$.

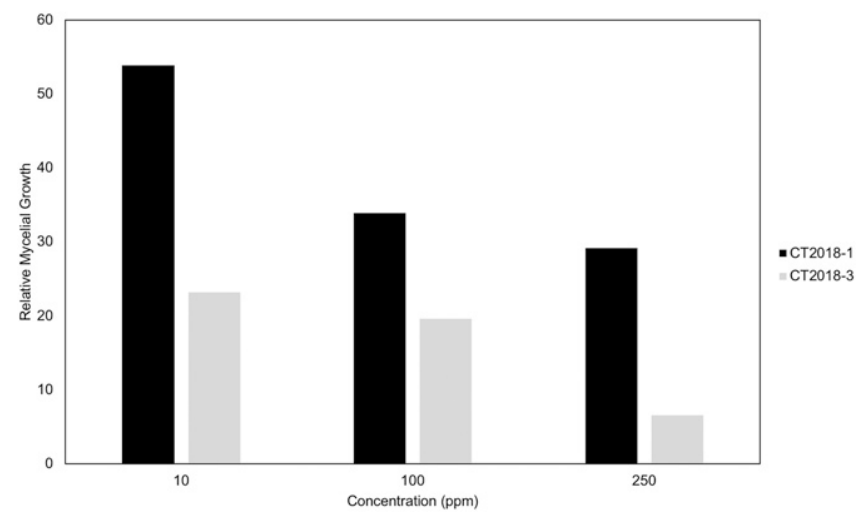

Fig. 5. Average relative mycelial growth of Diaporthe humulicola isolates CT2018-1 and CT2018-3 on trifloxystrobin fungicide-amended plates (representing two replicate experiments). humulicola has hyaline conidia and, based on our multigene tree, does not group with $S$. maydis. Within the family Diaporthaceae, phylogenetic analysis placed $D$. humulicola $98.4 \%$ support with $D$. ambigua, D. longispora, D. scerlotiodies, D. mayteni, D. raonikayaporum, Diaporthe sp. 2, D. angelica, D. subordinaria, D. arctii, D. neoarctii, D. cuppatea, D. lusitanicae, D. novem, D. infecunda, D. batatas, D. citri, D. sojae, Diaporthe sp. $1, D$. convolvuli, D. endophytica, D. phaseolorum, D. melonis, D. helianthin, D. hordei, D. vexans, D. megalospora, D. schini, D. tecomae, D. terebinthifolii, D. ganjae, D. manihota, D. oxe, Diaporthe sp. 3, D. paranensis, D. brasiliensis, and Diaporthe sp. 5 but was supported as its own taxa with 100\% support and distinct morphological features.

Many members of the genus Diaporthe (anamorph Phomopsis) are endophytes, saprobes, or pathogens of plant species worldwide (Gomes et al. 2013). Although some are host-specific pathogens, many have wide host ranges and they cause a variety of diseases, including cankers, leaf spots, dieback, rot, wilt, and blights (Gomes et al. 2013). Through Koch's postulates, it was demonstrated that D. humulicola causes leaf lesions on common hop. Field isolations showed that this organism was also present on cones. Based on the leaf spots characteristic of this disease, we propose the disease name of Diaporthe leaf spot.

The ability of $D$. humulicola to infect many hop cultivars and at two different locations shows the potential for this to become a major pathogen on hop in Connecticut. Weather conditions conducive for disease seem to be hot and humid weather, which often occurs in July and August in the northeastern United States. The early observance of this pathogen in May 2019, where it was not present in the spring in the same hop yards in 2018, may suggest that it overwintered in the

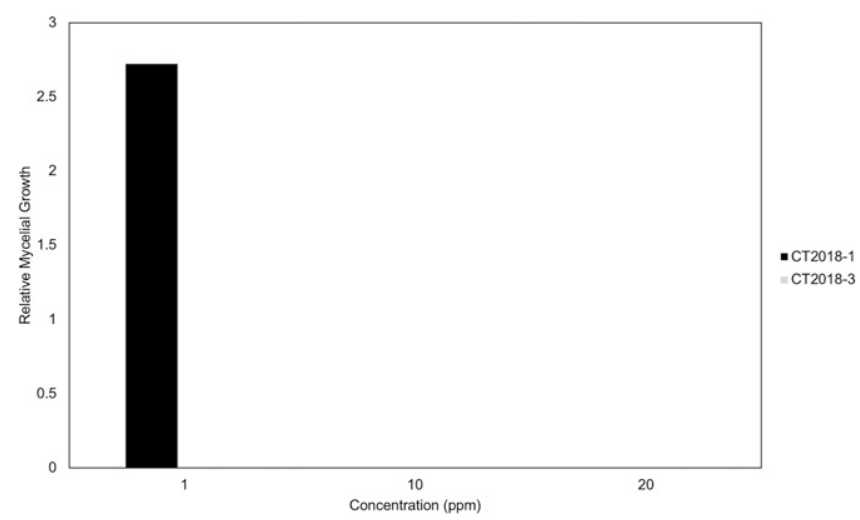

Fig. 6. Average relative mycelial growth of Diaporthe humulicola isolates CT2018-1 and CT2018-3 on pyraclostrobin and boscalid fungicide-amended plates (representing two replicate experiments).

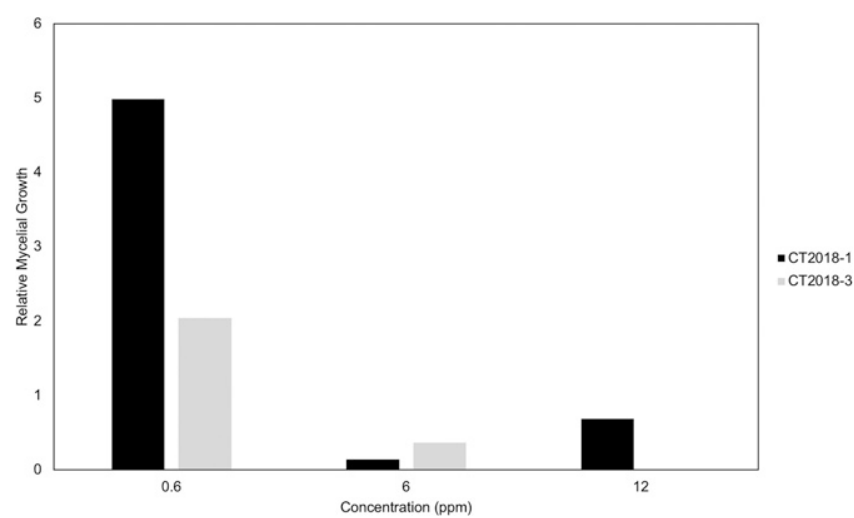

Fig. 7. Average relative mycelial growth of Diaporthe humulicola isolates CT2018-1 and CT2018-3 on pyraclostrobin fungicide-amended plates (representing two replicate experiments). 
hop yard and was able to cause infection early in the season. Fungicides applied when these weather conditions are predicted may reduce the severity of Diaporthe leaf spot. In vitro analysis of fungicide susceptibility indicated that pyraclostrobin, boscalid mixed with pyraclostrobin, and trifloxystrobin all reduce $D$. humulicola growth in culture. Future studies to determine the effective concentration for $50 \%$ inhibition of growth for each fungicide and the correlation of those with field efficacy need to be conducted in order to provide growers with fungicide recommendations. Further research is needed on alternative hosts and the disease cycle in order to develop management strategies for disease control. The high similarity of symptoms of Diaporthe leaf spot to Phoma wilt warrant further research into whether these two diseases are caused by D. humulicola and Phoma spp. or if they are caused by the same pathogen. The identification of D. humulicola in Connecticut is important to hop growers in the region because proper diagnosis and treatment may prevent hop cone loss. The spread of this pathogen into other hop-growing areas will need to be monitored as conducive weather conditions occur in hop-growing regions in the United States such as the Midwest. Although the Northeast is not a major hop-growing region, the movement of $D$. humulicola into the Midwest or Pacific Northwest could have large implications for hop cone production because the United States is the world's leader in supplying hop.

\section{Acknowledgments}

We thank J. Preste and R. Cecarelli for maintenance of hop yards and M. Salvas for assisting with field collections, culturing, and Koch's postulates.

\section{Literature Cited}

Allan-Perkins, E., Li, D. W., Schultes, N. P., Yavuz, S., and LaMondia, J. A. 2019a. First report of the resurgence of hop powdery mildew (Podosphaera macularis) in a New England commercial hop yard. Plant Dis. 103:1431.

Allan-Perkins, E., Maurer, K., and LaMondia, J. A. 2019b. Guidelines for integrated pest management for hops in Connecticut. CAES Bulletin 1057. https://portal.ct.gov/-/media/CAES/DOCUMENTS/Publications/Bulletins/B1057. pdf?la=en

Aveskamp, M. M., de Gruyter, J., Woudenberg, J. H. C., Verkley, G. J. M., and Crous, P. W. 2010. Highlights of the Didymellaceae: A polyphasic approach to characterize Phoma and related pleosporalean genera. Stud. Mycol. 65:1-60.

Aveskamp, M. M., Verkley, G. J., de Gruyter, J., Murace, M. A., Perello, A., Woudenberg, J. H., Groenewald, J. Z., and Crous, P. W. 2009. DNA phylogeny reveals polyphyly of Phoma section Peyronelleae and multiple taxonomic novelties. Mycologia 101:363-382.

Boerema, G. H., de Gruyter, J., Noordeloos, M. E., and Hamers, M. E. C., eds. 2004. Phoma Identification Manual: Differentiation of Specific and Infraspecific Taxa in Culture. CABI Publishing, Wallingford, U.K.

Carbone, I., and Kohn, L. M. 1999. A method for designing primer sets for speciation studies in filamentous ascomycetes. Mycologia 91:553-556.

Chi, P., Jiang, Z., and Xiang, M. 2007. Flora Fungorum Sinicorum: V 34 Phomopsis. Science Press, Beijing, China.

Crous, P. W., Groenewald, J. Z., Rised, J.-M., Simoneau, P., and Hywel-Jones, N. L. 2004. Calonectria species and their Cylindrocladium anamorphs: Species with sphaeropedunculate vesicles. Stud. Mycol. 50:415-430.

Crous, P. W., Slippers, B., Wingfield, M. J., Rheeder, J., Marasas, W. F. O., Philips, A. J. L., Alves, A., Burgess, T., Barber, P., and Groenewald, J. Z. 2006. Phylogenetic lineages in the Botryosphaeriaceae. Stud. Mycol. 55:235-253.

Crous, P. W., Wingfield, M. J., Schumacher, R. K., Summerell, B. A., Giraldo, A., Gené, J., Guarro, J., Wanasinghe, D. N., Hyde, K. D., Camporesi, E., Gareth Jones, E. B., Thambugala, K. M., Malysheva, E. F., Maylsheva, V. F., Acharya, K., Álvarez, J., Alvarado, P., Assefa, A., Barnes, C. W., Bartlett, J. S., Blanchette, R. A., Burgess, T. I., Carlavilla, J. R., Coetzee, M. P. A., Damm, U., Decock, C. A., den Breeÿan, A., de Vries, B., Dutta, A. K., Holdom, D. G., Rooney-Latham, S., Manjón, J. L., Marincowitz, S., Mirabolfathy, M., Morena, G., Nakashima, C., Papizedeh, M., Shahzadeh Fazeli, S. A., Swart, W. J., Tan, Y. P., Vanderbank, M., Wood, A. R., Zhang, Y., and Groenewald, J. Z. 2014. Fungal Planet description sheets: 281-319. Persoonia 33:212-289.

Dai, D.-Q., Wijayaywardene, N. N., Bhat, D. J., Chukeatirote, E., Zhao, R.-L., Wang, Y., Bahkali, A. H., and Hyde, K. D. 2014. The phylogenetic placement of Eriosporella babusicola sp. nov. in Capnodiales. Cryptogam. Mycol. 35:41-49.

Darby, H. 2017. 2016 Hop Pest Scouting Report. Northwest Crops and Soils Program, University of Vermont Extension. https://www.uvm.edu/sites/ default/files/Northwest-Crops-and-Soils-Program/2016-ResearchReports/2016_ HopPestScouting.pdf

Dissanayake, A., Camporesi, E., Hyde, K., Wei, Z., Yan, J., and Li, X. 2017. Molecular phylogenetic analysis reveals seven new Diaporthe species from Italy. Mycosphere 8:853-877.
Dissanayake, A. J., Liu, M., Zhang, W., Chen, Z., Udayanga, D., Chukeatirote, E. Li, X., Yan, J., and Hyde, K. 2015. Morphological and molecular characterization of Diaporthe species associated with grapevine trunk disease in China. Fungal Biol. 119:283-294.

Fan, X. L., Bezerra, J. D. P., Tian, C. M., and Crow, P. W. 2018. Families and genera of diaporthalean fungi associated with canker and dieback of tree hosts. Persoonia 40:119-134.

Fan, X. L., Tian, C. M., Qin, Y., Liang, Y. M., You, C. J., and Zhang, Y.-B. 2014 Cytospora from Salix in northern China. Mycotaxon 129:303-315.

Farr, D. F., and Rossman, A. Y. 2019. Fungal Databases. U.S. National Fungus Collections, USDA-ARS. https://nt.ars-grin.gov/fungaldatabases/

Fu, C.-H., Hseih, H.-M., Chen, C.-Y., Chang, T.-T., Huang, Y.-M., and Ju, Y.-M. 2013. Ophiodiaporthe cyathae gen. et sp. nov., a diaporthalean pathogen causing a devastating wilt disease of Cyathea lepifera in Taiwan. Mycologia 105:861-872.

Gao, Y., Liu, F., Duan, W., Crous, P. W., and Cai, L. 2017. Diaporthe is paraphyletic. IMA Fungus 8:153-187.

George, A. 2018. USA Hops 2017 Statistical Report. Hop Growers of America, Yakima, WA, U.S.A. https://www.usahops.org/

Gilman, J. C., and Archer, W. A. 1929. The fungi of Iowa parasitic on plants. Iowa State Coll. J. Sci. 3:299-507.

Glass, N. L., and Donaldson, G. C. 1995. Development of primer sets designed for use with the PCR to amplify conserved genes from filamentous ascomycetes. Appl. Environ. Microbiol. 61:1323-1330.

Gomes, R. R., Glienke, C., Videira, S. I. R., Lombard, L., Groenewald, J. Z., and Crous, P. W. 2013. Diaporthe: A genus of endophytic, saprophybic, and plant pathogenic fungi. Persoonia 31:1-41.

Gonzalez Fragoso, R. 1917. Fungi novi vel minus cognitarum horti botanici matritense. Trab. Mus. Nac. Ci. Nat. Ser. Bot. 12:1-99.

Grant, A., and Filotas, M. 2014. Downy Mildew in Ontario hops. Hort Matters, Ontario Ministry of Agriculture, Food, and Rural Affairs. http:// www.omafra.gov.on.ca/english/crops/hort/news/hortmatt/2014/11 hrt14a3.htm

Greene, H. C. 1944. Notes on Wisconsin Parasitic Fungi V. T. Wisc. Acad. Sci. 36: 225-268.

Groenewald, J. Z., Nakashima, C., Nishikawa, J., Shin, H.-D., Park, J.-H., Jama, A. N., Groenewald, M., Braun, U., and Crous, P. W. 2013. Species concepts in Cercospora: Spotting the weeds among the roses. Stud. Mycol. 75:115-170.

Guarnaccia, V., Groenewald, J. Z., Woodhall, J., Armengol, J., Cinelli, T., Eichmeier, A., Ezra, D., Fontaine, F., Gramaje, D., and GutierrezAguirregabiria, A. 2018. Diaporthe diversity and pathogenicity revealed from a broad survey of grapevine diseases in Europe. Persoonia 40:135-153.

Irinyi, L., János Kövichs, G., and Sánder, E. 2009. Taxonomical re-evaluation of Phoma-like soybean pathogenic fungi. Mycol. Res. 113:249-260.

Jakse, J., Radisek, S., Pokorn, T., Matousek, J., and Javornik, B. 2015. Deepsequencing revealed Citrus bark cracking viroid (CBCVd) as a highly aggressive pathogen on hop. Plant Pathol. 64:831-842.

Katoh, K., and Toh, K. 2008. Recent developments in the MAFFT multiple sequence alignment program. Brief. Bioinf. 9:286-298.

Kroon, L. P., Bakker, F. T., van den Bosch, G. B., Bonants, P. J., and Flier, W. G. 2004. Phylogenetic analysis of Phytophthora species based on mitochondrial and nuclear DNA sequences. Fungal Genet. Biol. 41:766-782.

Kumar, S., Stecher, G., and Tamura, K. 2016. MEGA7: Molecular Evolutionary Genetics Analysis version 7.0 for bigger datasets. Mol. Biol. Evol. 33:1870-1874.

Lamprecht, S. C., Crous, P. W., Groenewald, J. Z., Tewoldemedhin, Y. T., and Marasas, W. F. O. 2011. Diaporthaceae associated with root and crown rot of maize. IMA Fungus 2:13-24.

Li, D.-W., Schultes, N. P., Chen, J.-C., Wang, Y.-X., and Castañeda-Ruiz, R. F. 2017. Circinotrichum sinense, a new asexual fungus from Hubei, China Botany 95:1099-1108.

Liu, Y. J., Wheelen, S., and Hall, B. D. 1999. Phylogenetic relationships among ascomycetes: Evidence from an RNA polymerase II subunit. Mol. Biol. Evol 16:1799-1808

Mahaffee, W. F., Pethybridge, S. J., and Gent, D. H., eds. 2009. Pages 37-38 in: Compendium of Hop Diseases and Pests. American Phytopathological Society, St. Paul, MN, U.S.A.

Michel, V. V., Daepp, M., Woudenberg, J. H. C., de Gruyter, J., and de Wilt, P. J. G. M. 2018. First report of Boeremia exigua var. exigua causing stem and leaf spot on common speedwell in Switzerland. Plant Dis. 102:440.

Mulenko, W., Majewski, T., and Ruszkiewicz-Michalska, M. 2008. A preliminary checklist of Micromycetes in Poland. W. Szafer Institute of Botany, Polish Academy of Sciences 9:752.

MycoBank. 2019. MycoBank Database: Fungal Databases Nomenclature and Species Banks. http://www.mycobank.org/

O’Donnell, K., and Cigelnik, E. 1997. Two divergent intragenomic rDNA ITS2 types within a monophyletic lineage of the fungus Fusarium are nonorthologous. Mol. Phylogenet. Evol. 7:103-116.

Quaedvlieg, W., Groenewald, J. Z., de Jesús Yáñez-Morales, M., and Crous, P. W. 2012. DNA barcoding of Mycosphaerella species of quarantine importance to Europe. Persoonia 29:101-115.

Radisek, S., Jakse, J., Javornik, B., and de Gruyter, J. 2008. First report of Phoma exigua as a pathogen of hop in Slovenia. Plant Pathol. 57:381.

Rehner, S. A., and Samuels, G. J. 1994. Taxonomy and phylogeny of Gliocladium analyzed from nuclear large subunit ribosomal DNA sequences. Mycol. Res. 98:625-634. 
Ronquist, F., Teslenko, M., van der Mark, P., Ayres, D. L., Darling, A., Höhna, S., Larget, B., Liu, L., Suchard, M. A., and Huelsenbeck, J. P. 2012. MrBayes 3.2: Efficient Bayesian phylogenetic inference and model choice across a large model space. Syst. Biol. 61:539-542.

Stevens, J. F., and Page, J. E. 2004. Xanthohumol and related prenylflavonoids from hops and beer: To your good health! Phytochemistry 65:1317-1330.

Stöver, B. C., and Müller, K. F. 2010. TreeGraph 2: Combining and visualizing evidence from different phylogenetic analyses. BMC Bioinf. 11:7.

Sutton, B. C. 1980. The Coelomycetes. Fungi imperfecti with Pycnidia, Acervuli and Stromata. Commonwealth Mycological Institute, Kew, U.K.

Tan, Y. P., Edwards, J., Grice, K. R. E., and Shivas, R. G. 2013. Molecular phylogenetic analysis reveals six new species of Diaporthe from Australia. Fungal Divers. 61:251-260.

Thambugala, K. M., Daranagama, D. A., Phillips, A. J. L., Bulgakov, T. S., Bhat, D. J., Camporesi, E., Bahkali, A. H., Eungwanichayapant, P. D., Liu, Z.-Y., and Hyde, K. D. 2017. Microfungi on Tamarix. Fungal Divers. 82:239-306.

Udayanga, D., Castlebury, L. A., Rossman, A. Y., Chukeatirote, E., and Hyde, K. D. 2014a. Insights into the genus Diaporthe: Phylogenetic species delimitation in the D. eres species complex. Fungal Divers. 67:203-229.

Udayanga, D., Castlebury, L. A., Rossman, A. Y., Chaukeatirote, E., and Hyde, K. D. 2015. The Diaporthe sojae species complex: Phylogenetic reassessment of pathogens associated with soybean, cucurbits, and other field crops. Fungal Bio. 119:383-407.

Udayanga, D., Castlebury, L. A., Rossman, A. Y., and Hyde, K. D. 2014b. Species limits in Diaporthe: A molecular re-assessment of D. citri, D. cytosporella, D. foeniculina, and D. rudis. Persoonia 32:83-101.

Udayanga, D., Liu, X., Crous, P. W., McKenzie, E. H. C., Chaukeatirote, E., and Hyde, K. D. 2012. A multi-locus phylogenetic evaluation of Diaporthe (Phomopsis). Fungal Divers. 56:157-171.

Udayanga, D., Liu, X., McKenzie, E. H. C., Chaukeatirote, E., Bahkali, A. H. A., and Hyde, K. D. 2011. The genus Phomopsis: Biology, applications, species concepts and names of common phytopathogens. Fungal Divers. 50:189-225.

USDA. 1960. Index of Plant Diseases in the United States. USDA Agric. Handb. 165. U.S. Department of Agriculture, Washington, DC, U.S.A.

Van den Ende, A., and de Hoog, G. 1999. Variability and molecular diagnostics of the neurotropic species Cladophialophora bantiana. Stud. Mycol. 43:151-162.
Vilgalys, R., and Hester, M. 1990. Rapid genetic identification and mapping of enzymatically amplified ribosomal DNA from several Cryptococcus species. J. Bacteriol. 172:4238-4246.

Villesen, P. 2007. FaBox: An online toolbox for FASTA sequences. Mol. Ecol Notes 7:965-968.

Vu, D., Groenewald, M., de Vries, M., Gehrmann, T., Stielow, B., Eberhardt, U., Al-Hatmi, A., Groenewald, J. Z., Carinali, G., Houbraken, J., Boekhout, T. Crous, P. W., Robert, V., and Verkley, G. J. M. 2019. Large-scale generation and analysis of filamentous fungal DNA barcodes boosts coverage for kingdom fungi and reveals thresholds for fungal species and higher taxon delimitation. Stud. Mycol. 92:135-154.

Wanasinghe, D. N., Phukhamsakda, C., Hyde, K. D., Jeewon, R., Lee, H. B., Jones, G. E. B., Tibpromma, S., Tennakoon, D. S., Dissanayake, A. J., Jayasiri, S. C., Gafforov, Y., Camporesi, E., Bulgakov, T. S., Ekanayake, A. H., Perera, R. H. Samarakoon, M. C., Goonasekara, I. D., Mapook, A., Li, W.-J., Senanayake, I. C., Li, J., Norphanphoun, C., Doilom, M., Bahkali, A. H., Xu, J., Mortimer, P. E., Tibell, L., Savic, S. T., and Karunarathna, S. C. 2018. Fungal diversity notes 709-839: Taxonomic and phylogenetic contributions to fungal taxa with an emphasis on fungi on Rosaceae. Fungal Divers. 89:1-236.

White, T. J., Bruns, T. D., Lee, S. B., and Taylor, J. W. 1990. Amplification and direct sequencing of fungal ribosomal RNA genes for phylogenetics. Pages 315-322 in: PCR Protocols: A Guide to Methods and Applications, Vol. 18 M. A. Innis, D. H. Gelfand, J. J. Sninsky, and T. J. White, eds. Academic Press, Inc., New York, NY, U.S.A.

Yang, Q., Fan, X.-L., Du, Z., and Tian, C.-M. 2017. Diaporthe species occurring on Senna bicapsularis in southern China, with descriptions of two new species. Phytotaxa 302:145-155.

Yang, Q., Fan, X.-L., Du, Z., and Tian, C.-M. 2018a. Diaporthosporellaceae, a novel family of Diaporthales (Sordariomycetes, Ascomycota). Mycoscience 59:229-235

Yang, Q., Fan, X.-L., Guarnaccia, V., and Tian, C.-M. 2018b. High diversity of Diaporthe species associated with dieback diseases in China, with twelve new species described. MycoKeys 39:97-149.

Zanoli, P., and Zavatti, M. 2008. Pharmacognostic and pharmacological profile of Humulus lupulus L. J. Ethnopharmacol. 116:383-396.

Zhuang, W.-Y. 2005. Fungi of Northwestern China. Mycotaxon, Ltd., Ithaca, NY, U.S.A. 